\title{
NEAT in der Schweiz - Versprechungen gehalten? Lehren aus einer über 30-jährigen Geschichte
}

\author{
Heinz Ehrbar
}

\section{Kurzfassung}

Mit der Inbetriebnahme des Ceneri-Basistunnels per Ende 2020 ging die erste Etappe des Baus der Neuen Eisenbahn-Alpentransversale (NEAT) in der Schweiz nach beinahe 30 Jahren zu Ende. Im Jahr 1992 wurde mit der Volksabstimmung über den Alpentransit-Beschluss des schweizerischen Parlaments der Grundstein zum Bau der zwei Achsen am Lötschberg und am Gotthard geschaffen. Die Arbeiten an einem für schweizerische Verhältnisse gigantischen Ausbauschritt des Eisenbahnsystems begannen und sollten gemäss Zielvorgabe aus dem Jahr 1992 den überwiegenden Teil des alpenquerenden Gütertransitverkehrs durch die Schweiz von der Strasse auf die Schiene verlagern. Die Fahrzeiten zwischen Nord und Südschweiz sollten massiv verkürzt werden. Mit der NEAT war zudem beabsichtigt die Schweiz im Norden und im Süden an das künftige moderne Schienennetz Europas anzuschliessen. Die dafür zu tätigenden Investitionen in die neuen Eisenbahnlinien am Lötschberg und am Gotthard sollten aber auch der der Umwelt und der Wirtschaft dienen.

Zum jetzigen Zeitpunkt der Vollendung der ersten NEAT-Ausbauetappe stellt sich nun zu Recht die Frage, ob diese Ziele erreicht wurden oder aber in naher Zukunft noch erreicht werden. Der nachfolgende Beitrag soll darüber Auskunft geben.

\footnotetext{
Article note: Der Autor bedankt sich bei den Herren Peter Zbinden, ehemaliger CEO Alptransit Gotthard AG und Hans-Peter Vetsch, Vetsch Rail-Consulting GmbH (ehemals AlpTransit Gotthard AG) für die wertvolle Unterstützung bei der Erarbeitung dieses Beitrags.
}

H. Ehrbar $(\bowtie)$

Institut für Bau- und Infrastrukturmanagement, ETH Zürich, Zürich, Schweiz

E-Mail: hehrbar@ethz.ch

S. Laimer, C. Perathoner (Hrsg.), Mobilitäts- und Transportrecht in Europa,

Bibliothek des Wirtschaftsrechts 2,

https://doi.org/10.1007/978-3-662-63635-0_10 


\section{Die Entstehung des Projekts AlpTransit}

Bis ins Jahr 400 n.Chr. bauten die Römer erste Verkehrswege über die Alpen. Seither war das Gebiet der heutigen Schweiz immer ein Transitland im Herzen Europas. Die von den Römern nicht bezwingbare Schöllenenschlucht im Gotthardgebiet wurde anfangs des 13. Jahrhunderts ein erstes Mal mit einem Saumpfad ausgebaut. In den darauffolgenden Jahrhunderten erfolgte der schrittweise Ausbau zu einer Passstrasse, welche 1830 ein europaweit anerkannt hohes Qualitätsniveau erreichte. Mit der Eröffnung des Gotthard-Eisenbahntunnels 1882 übernahm dann die Bahn für die nächsten Jahrzehnte die Lasten des Personen- und Güterverkehrs für den alpenquerenden Verkehr, welcher bisher durch Säumer und Postkutschen über die Passstrasse bewältigt wurde. Seit 1913 stand mit der Lötschberg-Simplon Linie eine zweite Achse zur Verfügung (Abb. 1).

Die Anforderungen an die Verkehrsinfrastruktur in den Alpen änderten sich mit dem Aufkommen des Automobils nach dem zweiten Weltkrieg. Bereits ab dem Ende der fünfziger Jahre des letzten Jahrhunderts stauten sich in den Sommermonaten die Autos am Gotthard (Abb. 2 links), während in den Wintermonaten Totenstille herrschte. Es gab damals keine im Winter befahrbaren Passstrassen zwischen der Deutschschweiz und dem italienisch sprechenden Tessin (Abb. 2 rechts). Einzig die Gotthard-Eisenbahn verband die beiden Landesteile. Im Zuge des Aufbaus des Autobahnnetzes ab 1960 erklang dementsprechend der Ruf nach einer wintersicheren Strassenverbindung zwischen dem Norden und dem Süden. Eine der untersuchten Varianten sah vor, den bestehenden Eisenbahntunnel in einen Strassentunnel umzubauen und die Bahn in einen neu zu erstellenden Basistunnel von Amsteg nach Bodio zu verlegen. 1963 wurde dann der Bau eines 16,4 km langen Strassentunnels zwischen Göschenen und Airolo beschlossen, allerdings mit der

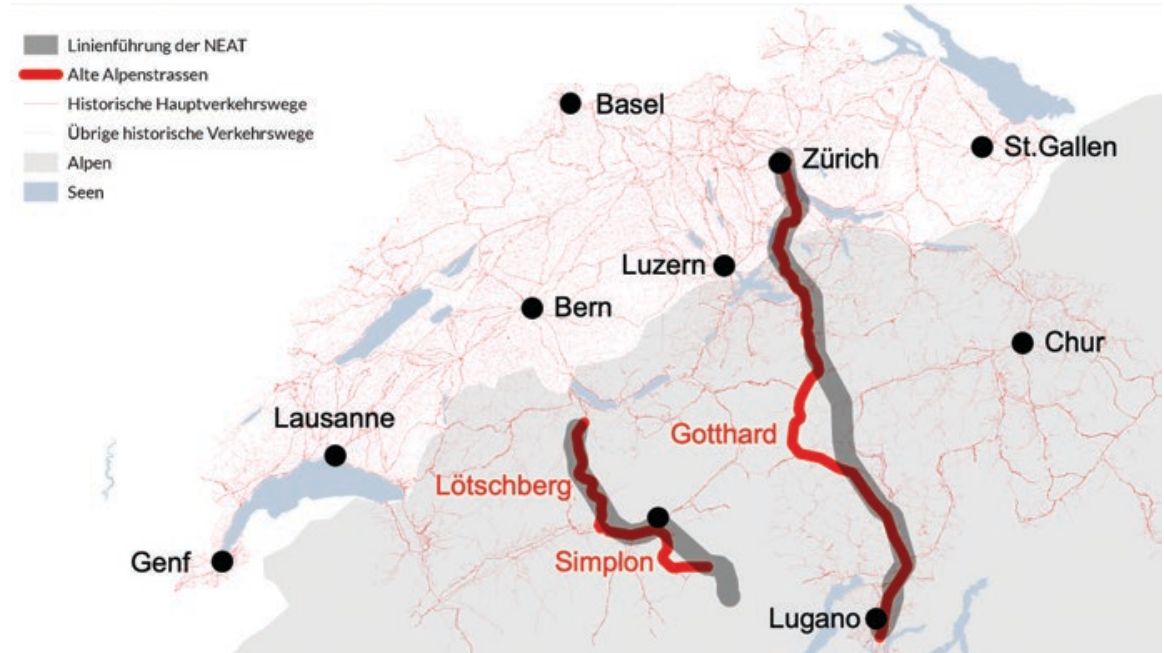

Abb. 1 Historische Hauptverkehrswege und die alpenquerenden Korridore am Gotthard und Lötschberg - Simplon (Hintergrundbild: https://www.alptransit-portal.ch/en/media/file/1599/) 


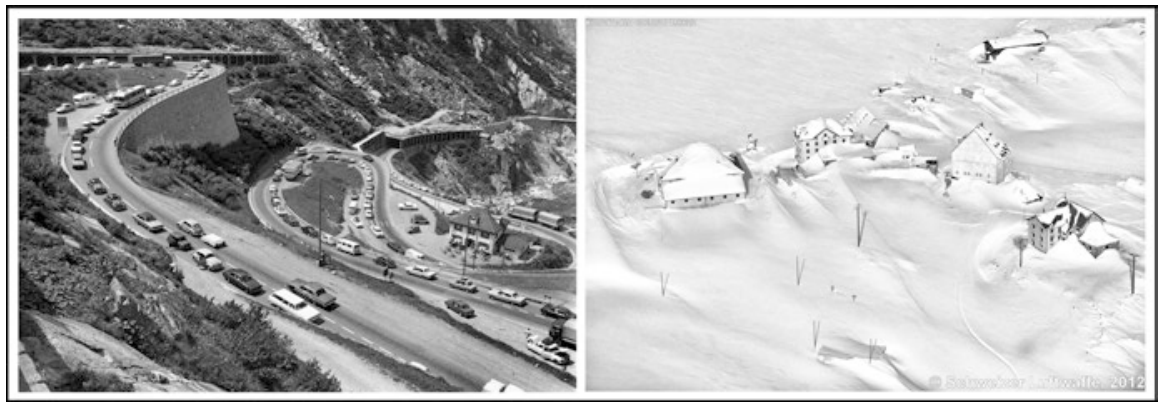

Abb. 2 Strassenverkehr am Gotthard (Tremola) im Sommer und Gotthard-Hospiz im Winter (Bildquellen: links: Agentur Keystone, rechts: Schweizer Luftwaffe, Copyright VBS)

Auflage, dass das Projekt des Eisenbahn-Basistunnels dann verfolgt werden solle, wenn dies für den zukünftigen Verkehr erforderlich wäre.

Die entsprechenden Vorabklärungen wurden durch die breit abgestützte eidgenössische Kommission Eisenbahntunnel durch die Alpen (KEA) sofort an die Hand genommen. In ihrem Schlussbericht schlug die Kommission im Jahr 1971 den sofortigen Ausbau der Lötschberglinie auf Doppelspur als notwendige Übergangslösung bis zum Bau eines Gotthard-Basistunnels, aber auch zum GotthardBasistunnel wurde folgende Aussage getätigt: „Angesichts des sämtliche Prognosen übersteigenden internationalen Transitverkehrs sollte mit dem Bau möglichst rasch begonnen werden. " 1 Begründet wurden diese Empfehlungen mit der Vorrangstellung der Gotthardroute im europäischen Verkehr seit historischen Zeiten bis in die Gegenwart.

Geprägt durch die Hochkonjunktur der Sechziger-Jahre ging man gleich ans Werk und die Schweizerischen Bundesbahnen (SBB) wurden beauftragt ein Bauprojekt (Entwurfsplanung) für einen Basistunnel auszuarbeiten. Die wirtschaftlichen Rahmenbedingungen trübten sich in der Schweiz aber spätestens ab 1973 (Ölkrise) rasch ein. Der Verkehrseinbruch traf vor allem die schweizerischen Bahnen, ganz im Gegensatz zum benachbarten Ausland und zum LKW-Verkehr durch die schweizerischen Alpen, wo das Transportvolumen trotz wirtschaftlichem Rückgang zunahm (Abb. 3).

Damit verflog dann auch die Euphorie für den Bau eines Basistunnels bei den politischen Entscheidungsträgern. Der schweizerische Bundesrat (Exekutive) beschloss deshalb 1975, das von den SBB vorgelegte Bauprojekt mit einem rund 46 km langen Doppelspurtunnel ${ }^{2}$ (Abb. 4) nicht zu realisieren.

\footnotetext{
${ }^{1}$ Eisenbahntunnel durch die Alpen, Kommission des Eidgenössischen Verkehrs- und Energiewirtschaftsdepartements, Schlussbericht 1971.

${ }^{2}$ Rutschmann, Werner; Neue Eisenbahn-Alpentransversale Gotthard-Basislinie, Opfer der Politik und des Kleinmuts, SBB historic, 2004.
} 


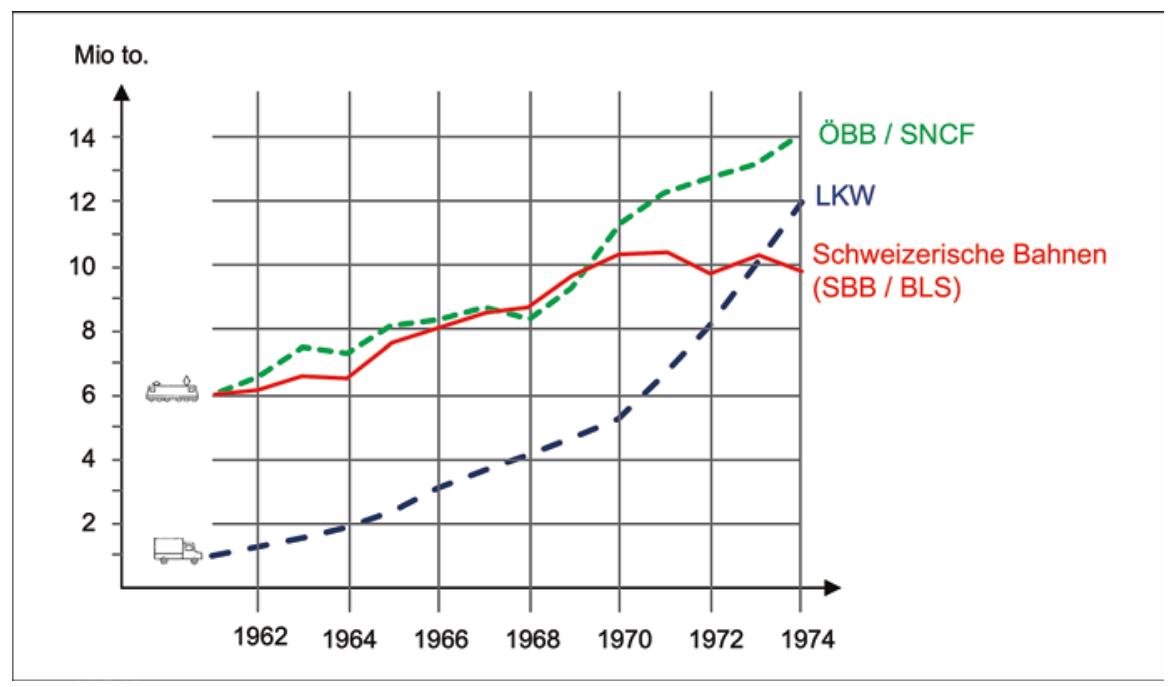

Abb. 3 Entwicklung des alpenquerenden Verkehrs (1961-1974) (Datenquelle ( ${ }^{B}$ otschaft des Bundesrates an die Bundesversammlung über den Ausbau der Bern-Lötschberg-Simplon-Bahn auf Doppelspur vom 4. Februar 1976.))

Als teilweise Kompensation wurde der zweigleisige Ausbau der bisher eingleisigen Rampen auf der Nord- und der Südseite der Lötschbergbahn beantragt ${ }^{3}$ und vom Parlament in Auftrag ${ }^{3}$ gegeben.

In der Zeit von 1972 bis 1980 wurde dann der Gotthard-Strassentunnel unter teilweise schwierigen Randbedingungen gebaut. Mit seiner Eröffnung im September 1980 gab der damals für den Straßenverkehr zuständige Innenminister, Bundesrat Hürlimann das Versprechen ab, dass dieser Tunnel kein Korridor für den Schwerverkehr sein werde ${ }^{4}$. Die daraufhin einsetzende Verkehrsentwicklung zeigte jedoch rasch, dass dieses Versprechen nicht zu halten war. Der Güterverkehr auf der Strasse nahm enorm zu, während er beim Schienenverkehr zurückging. Den SBB drohte eine wichtige Einnahmenquelle wegzubrechen.

Parallel dazu wurden mit Lärm, Staub, Abgasen und Unfallereignissen auch die negativen Folgen des Schwerverkehrs auf den Strassen in den engen Alpentälern erkennbar. Der Ruf nach Gegenmassnahmen erklang, wobei sich dieser nicht nur auf die Schweiz beschränkte. Auch Österreich litt (und leidet) an der Brennerachse unter den gleich schwierigen Verhältnissen. So lag es denn ab Mitte der AchtzigerJahre nahe, den Ausbau der Verkehrsachsen durch die Alpen international abzustimmen. 1988 wurde bei einem alpenquerenden Gütervolumen von rund 68 Mio. Tonnen die Prognose getätigt, dass bis 2020 das Gütertransitvolumen durch

\footnotetext{
${ }^{3}$ Bundesbeschluss über den Ausbau der Bern-Lötschberg-Simplon-Bahn auf Doppelspur vom 22. Juni 1976

${ }^{4}$ Manuskript der Rede von Bundesrat Hürlimann vom 5. September 1980.
} 

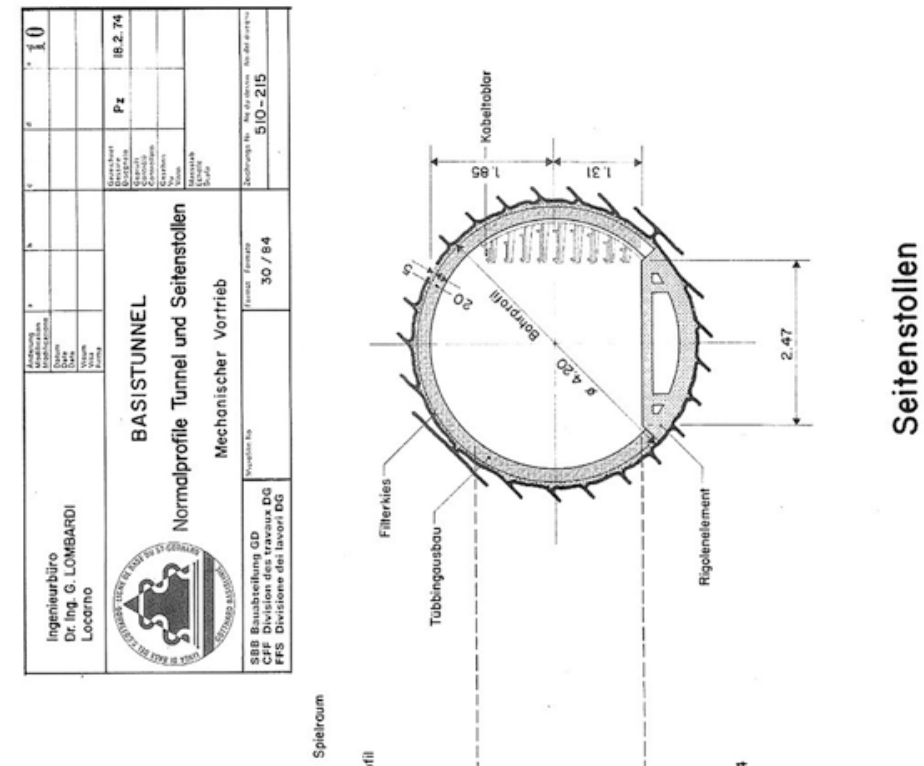

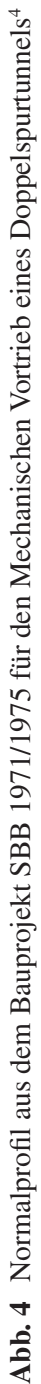


die Alpen auf rund 140 Mio. Tonnen steigen würde. ${ }^{5}$ Die Länder Österreich, Schweiz, Frankreich sollten auf ihren Transitkorridoren je ein Drittel des Transitverkehrs nach Italien übernehmen (Abb. 5).

Die Schweiz betonte dabei immer, dass sie nur bereit war diesen Mehrverkehr ausschliesslich auf der Schiene zu übernehmen; eine Position, welche damals auch von den Nachbarländern übernommen wurde.

Der Ausschuss der stellvertretenden Verkehrsminister Deutschlands, Österreichs, Italiens und der Schweiz kam demzufolge 1989 zum Schluss, dass sich die Strassenverkehrskapazitäten über die Alpen kaum mehr erhöhen lassen würden und dass demzufolge der prognostizierte künftige Neuverkehr auf die Schiene zu verlagern wäre. Dazu brauche es zwei neue Schienenverkehrsachsen durch die Alpen, eine am Brenner und eine andere durch die Schweiz ${ }^{6}$ (Abb. 6).

Die schweizerische Bundesregierung oblag bei dieser Ausgangslage die Aufgabe, die seit Jahrzehnten umstrittene Linienführung (Ostalpenbahn (Splügen), Gotthard (Ost, Mitte, West), Ypsilon-Variante, Lötschberg - Simplon) durch die Schweiz festzulegen. Aus dem Beteiligungsverfahren unter den Kantonen (Vernehmlassungsverfahren) zeigte sich, dass keine der singulären Lösungen mehrheitsfähig war. Nur eine Netzlösung mit dem gleichzeitigen Bau von zwei Achsen am Lötschberg und am Gotthard fand Zustimmung bei den Kantonen, welche ein Potenzial von mehr als $50 \%$ der Gesamtbevölkerung repräsentierten.

Diese Netzlösung (Abb. 7) wurde mit der Botschaft über den Bau der schweizerischen Eisenbahn-Alpentransversale (Alpentransit-Beschluss) vom 23. Mai 1990
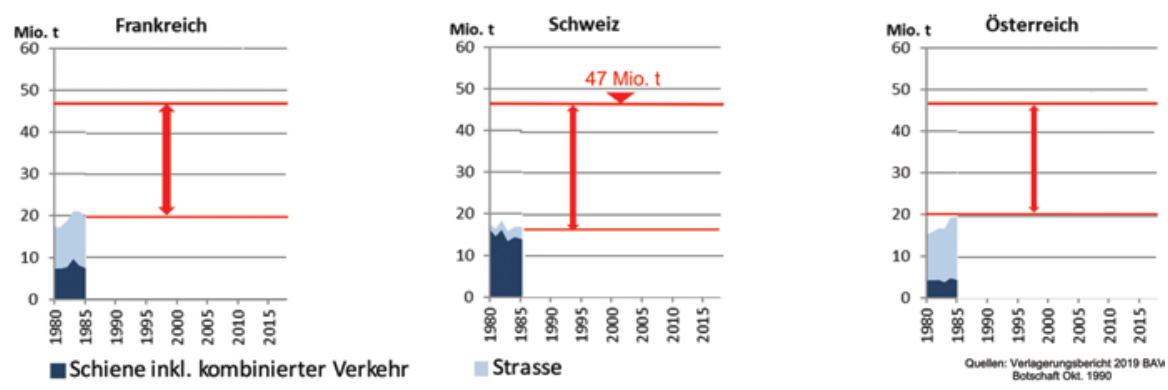

Transalpiner Güterverkehr 1984 (total):

ca. 68 Mio. $t$

Prognose für 2020: 140 Mio. $t \quad \Rightarrow \quad$ Jedes Land soll ein Drittel tragen

Abb. 5 Generelle Planungshypothese für den Ausbau der Transitkapazitäten durch die Alpen

\footnotetext{
${ }^{5}$ Schweizerischer Bundesrat, Botschaft über den Bau der schweizerischen Eisenbahn-Alpentransversale (Alpentransit-Beschluss) vom 23. Mai 1990.

${ }^{6}$ Verkehrsminister der Bundesrepublik Deutschland, Italiens, Österreichs und der Schweiz, Verbesserung des alpenquerenden Verkehrs, Schlussbericht des Stellvertreter-Ausschusses vom April 1989.
} 


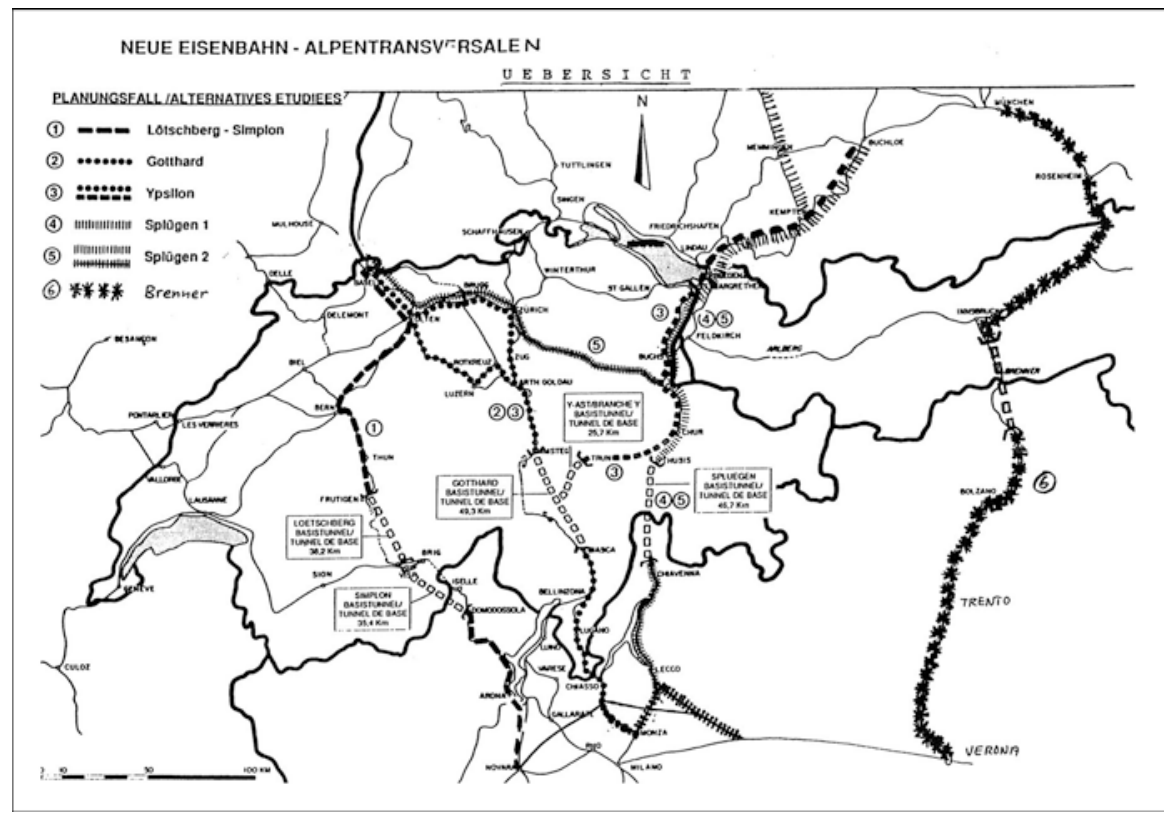

Abb. 6 Im Rahmen des Stellvertreter-Ausschusses berücksichtigte mögliche Varianten ${ }^{8}$

dem Parlament zum Beschluss vorgelegt, von diesem im Oktober 1990 debattiert und mit einer überwiegenden Mehrheit genehmigt.

Die grüne Partei und die Automobil-Lobby machten daraufhin von den direktdemokratischen Rechten Gebrauch und erzwangen mit einem äusserst knapp zustande gekommenen Referendum eine Volksabstimmung über den entsprechenden Bundesbeschluss. Am 27. September 1992 wurde dem Projekt mit 63,3 \% Ja-Stimmenanteil und mit Ja-Mehrheiten in 21 von 23 Kantonen (nur der direkt betroffene Kanton Uri und die beiden Appenzeller Halbkantone sagten nein) zugestimmt (Abb. 8). Das NEAT-Projekt war geboren.

\section{Wem wurde wann was versprochen?}

Infrastrukturprojekte müssen einen auf das jeweilige Projekt zugeschnittenen Anforderungskatalog erfüllen, welcher sich an die Qualitäts-, Termin- und Kostenzielen zu orientieren hat. Unter die Qualitätsziele fallen alle Anforderungen den bautechnischen Eigenschaften, zur Funktionalität sowie die Anforderungen des vom Projekt betroffenen Umfeldes. Dabei sind viele Interessenspartner bei der Formulierung der Anforderungen zu berücksichtigen (Abb. 9). 


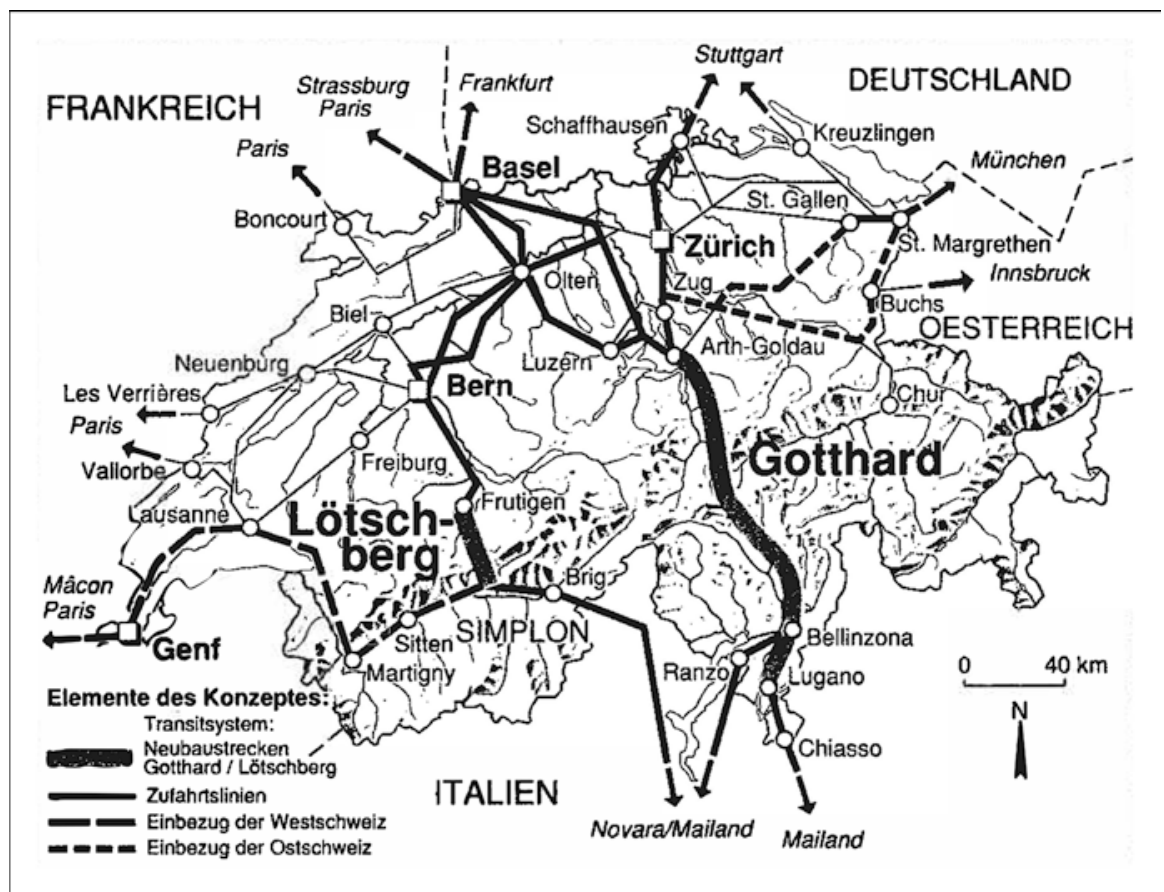

Abb. 7 Die NEAT Netzlösung gemäss Konzept des schweizerischen Bundesrats 1990 (Schweizerische Eidgenossenschaft, Volksabstimmung vom 27. September 1992, Erläuterungen des Bundesrates.)

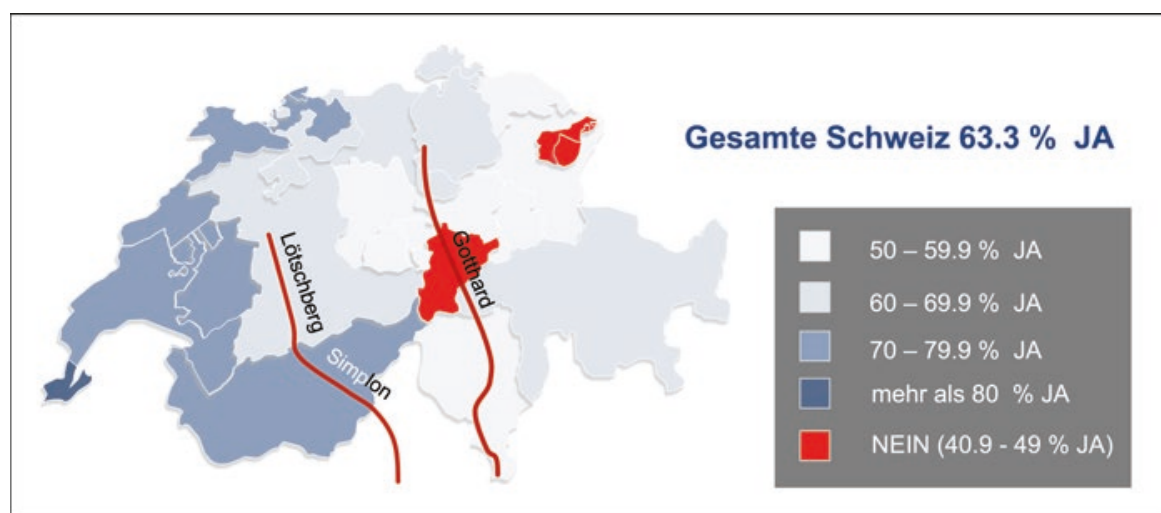

Abb. 8 Abstimmungsergebnis vom 27. September 1992 über den Alpentransit-Beschluss 


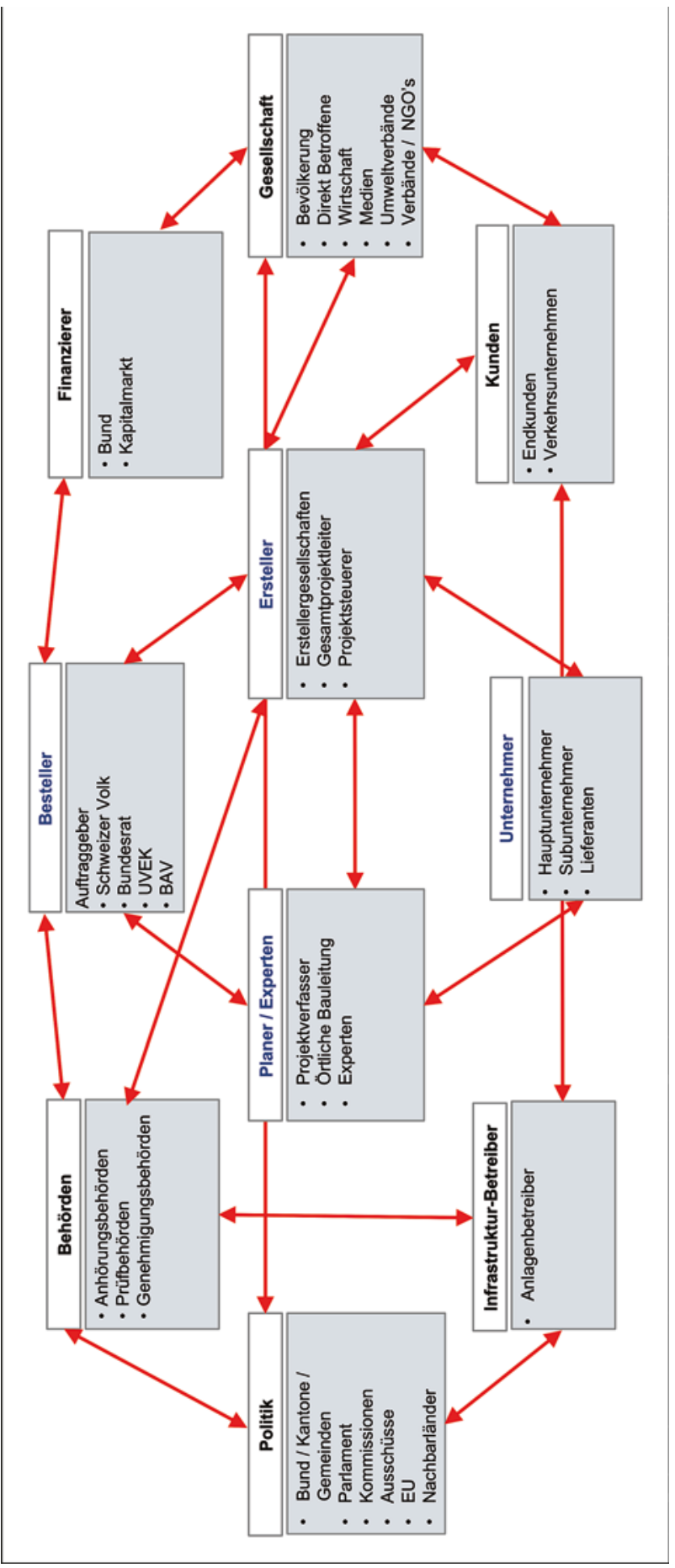

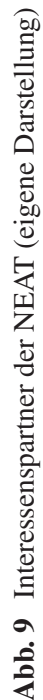


Bei der NEAT wurden die Anforderungskataloge jeweils in den Botschaften an das Parlament und in den Erläuterungen des Schweizerischen Bundesrates im Hinblick auf die Volksabstimmungen veröffentlicht. Ausgangspunkt der nachfolgenden Betrachtungen sind deshalb diese Dokumente (vgl. Fussnoten).

Direkter Adressat der Aussagen in den Botschaften waren die Parlamentarier und mit den Abstimmungserläuterungen jeder einzelne Stimmbürger. In den Abstimmungsempfehlungen für die erste Abstimmung über den Alpentransitbeschluss vom September 1992 wurden zusätzlich verschiedene Interessensgruppierungen (Stakeholder) direkt angesprochen, wie z. B. die Bahnkunden (Personen- und Güterverkehr), die Automobilisten, die Bahngesellschaften, die vom Projekt betroffenen Regionen, die Nachbarländer und die Europäische Union (EU, damals EG).

\section{1 Übergeordnete Zielsetzung der NEAT}

1. Der überwiegende Teil des Gütertransitverkehrs soll von der Strasse auf die Schiene verlagert werden.

2. Die Fahrzeiten sollen zwischen der Nord und der Südschweiz halbiert werden.

3. Der Anschluss der Schweiz an das künftige moderne Schienennetz Europas soll sichergestellt werden.

4. Die Investitionen (14,9 Mrd. Franken, Projekt- und Preisstand 1991) sollen der Umwelt und der Wirtschaft dienen. ${ }^{7}$

\subsection{Im Jahr 1992 vorgesehener Projektumfang}

Das Vorhaben der neuen schweizerischen Eisenbahn-Alpentransversale (NEAT) umfasste gemäss der Abstimmungsvorlage vom September $1992^{10}$ die folgenden vier Elemente (Abb. 7):

a) Die Neubaustrecke Arth-Goldau/Lugano mit Basistunneln am Gotthard (ca. $50 \mathrm{~km}$ ) und am Monte Ceneri $(12,6 \mathrm{~km})$, welche die Südschweiz wesentlich besser an die Nord-schweiz anbindet

b) Die Basislinie aus dem Raum Frutigen ins Rhonetal (ca. $30 \mathrm{~km}$ ). Diese verbindet zudem die zwei Nationalstrassen A6 (Bern-Thun-Spiez) und A9 (Wallis), indem sie als Ersatz für die Rawilstrecke auch dem Autoverlad dient und das Wallis besser erschliesst

c) Die Schweiz wird über Genf und Basel an das europäische Hochgeschwindigkeitsnetz angeschlossen, wobei die zwischen Genf und Basel liegenden Ver-

\footnotetext{
${ }^{7}$ Schweizerische Eidgenossenschaft, Volksabstimmung vom 27. September 1992, Erläuterungen des Bundesrates.
} 
bindungen im Jura verbessert werden um damit den engeren Anschluss der Westschweiz an das europäische Schienennetz gewährleisten.

d) Die Ostschweiz wird durch den Bau neuer Linien aus dem Raum Zug in den Raum Zürichsee in das Gesamtkonzept eimbezogen.

Darüber hinaus wurde in Aussicht gestellt, die Zufahrten von St. Gallen in den Raum Zürichsee zu verbessern und den Bahnhof Chur auszubauen. In der Surselva waren verbesserte Eisenbahnzufahrten zur Erschliessung des geplanten Zwischenangriffs in Sedrun vorgesehen.

\subsection{Nutzen für den Güterverkehr}

Die Schweiz, mitten in Europa, hatte und hat stets einen beträchtlichen Teil des europäischen Transitgüterverkehrs zwischen Norden und Süden zu tragen. Wegen der lange fehlenden Strassenkapazitäten und dank der gut ausgebauten Schieneninfrastruktur wurden im Jahr 199285 Prozent des Transitgüterverkehrs auf der Schiene und nur 15 Prozent auf der Strasse abgewickelt ${ }^{10}$.

Um den prognostizierten zusätzlichen Verkehr auch künftig mit der Bahn umweltfreundlich bewältigen zu können, brauchte es einen Ausbau der Schienenverkehrskapazitäten. Mit der NEAT, welche in der Lage sein sollte den gesamten künftig auf die schweizerischen Nord- und Südgrenzen zurollende Gütertransitverkehr per Bahn zu transportieren, wurde der entsprechende Ausbauschritt für das Schienennetz in die Wege geleitet. Ohne den Ausbau des Schienennetzes wäre langfristig bei vollkommener Verlagerung des Güterverkehrs auf die Strasse (bei Berücksichtigung der 28 Tonnen-Limite in der Schweiz, Nachtfahrverbot für Lastkraftwagen (LKW)) mit 7 bis 9 Millionen Lastwagendurchfahrten pro Jahr durch die Schweizer Alpen zu rechnen gewesen. ${ }^{8}$ Eine solche Verkehrslawine hätte unweigerlich enorme Staus auf den Transitrouten ausgelöst, welche auch den privaten Personenverkehr stark beeinträchtigt hätten.

Somit ergab sich das Ziel, dass dank der NEAT der gesamte künftige Mehrverkehr auf modernen, im Schnellzugstempo verkehrenden Zügen des kombinierten Verkehrs zwischen den nordeuropäischen Ballungsräumen und den Wirtschaftszentren Italiens befördert werden kann. Dieses Verlagerungsziel setzte voraus, dass in den betroffenen Ländern geeignete Verladekapazitäten für Strassenfahrzeuge oder von Teilen derselben (Sattelauflieger, Wechselaufbauten) geschaffen werden.

Ohne Zwangsmassnahmen geht eine solche Verlagerung von der Strasse auf die Schiene aber (Abb. 10) nur dann, wenn das Angebot der Bahn qualitativ (Pünktlichkeit, Kapazität, Frequenz) einwandfrei ist. Eine hohe Transportqualität setzt eine ausreichend ausgebaute Infrastruktur voraus (Netzausbauten plus Verladeterminals) aber auch optimierte betriebliche Abläufe im grenzüberschreitenden Verkehr

\footnotetext{
${ }^{8}$ Schweizerische Eidgenossenschaft, Volksabstimmung vom 27. September 1992, Erläuterungen des Bundesrates.
} 


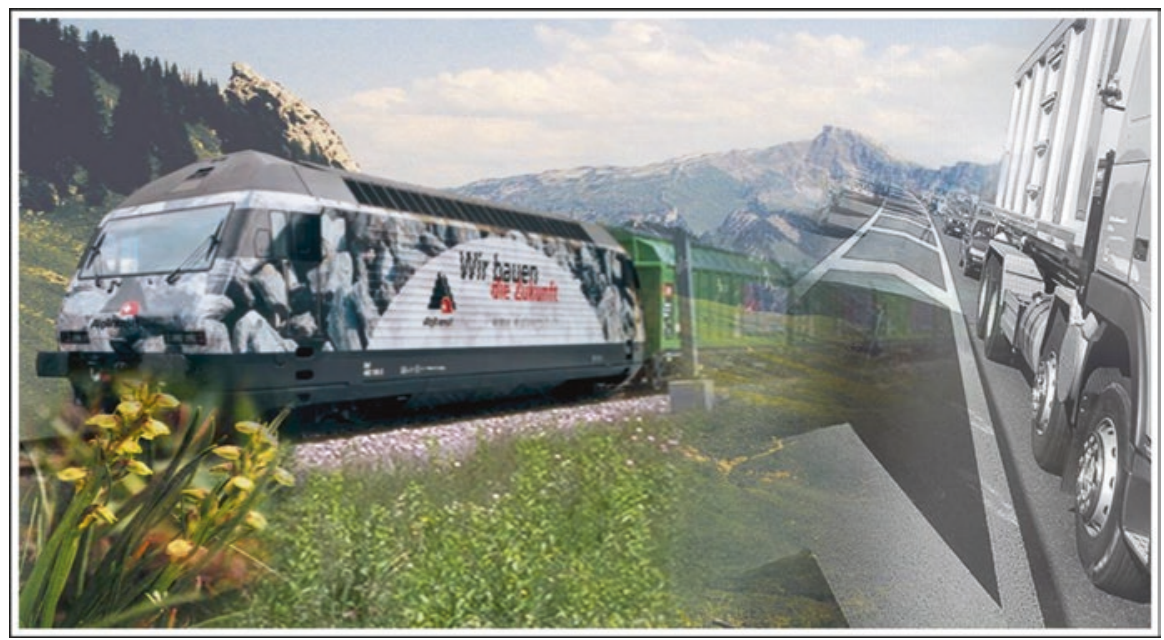

Abb. 10 Primäres Ziel der NEAT: Verlagerung des Güterverkehrs auf die Schiene (Bild AlpTransit Gotthard AG)

(z. B. über die Einführung einer europaweit standardisierten Leit- und Sicherungstechnik). Die NEAT hatte dafür zu sorgen, dass diese Voraussetzungen erfüllt werden konnten.

\subsection{Nutzen für den Personenverkehr}

Hauptziel im Personenverkehr war die wesentlich engere Verknüpfung der nördlichen und der südlichen Landesteile, was zur generellen Zielsetzung einer Halbierung der Fahrzeiten zwischen den Zentren beidseits der Alpen führte. Zürich oder Luzern sollten mit Bellinzona in gut einer Stunde verbunden werden. Von Bern sollte man in etwas weniger als einer Stunde nach Brig reisen oder aber in weniger als 2 Stunden an den Lago Maggiore fahren können. Neuenburg wäre dank der NEAT noch knapp $13 / 4$ Stunden vom Wallis entfernt. Durch wesentlich bessere Umsteigebeziehungen sollten auch Reiseziele ausserhalb der Städte und Agglomerationen in kürzerer Zeit erreicht werden. ${ }^{9}$

Aber auch im internationalen Fernverkehr, sollte die NEAT ihren Nutzen entfalten. Die Nachbarländer Frankreich, Deutschland und Italien unternahmen seit den Achtziger-Jahren grosse Anstrengungen, um das Eisenbahnnetz mit Neubaustrecken für den Hochgeschwindigkeitsverkehr attraktiv zu gestalten und dank kurzen Fahrzeiten der neuen Züge eine echte Alternative zum Flugzeug über Distanzen von 500 bis $1000 \mathrm{~km}$ zu bieten. Die Schweiz hatte somit alles Interesse daran, den

\footnotetext{
${ }^{9}$ Schweizerische Eidgenossenschaft, Volksabstimmung vom 27. September 1992, Erläuterungen des Bundesrates.
} 


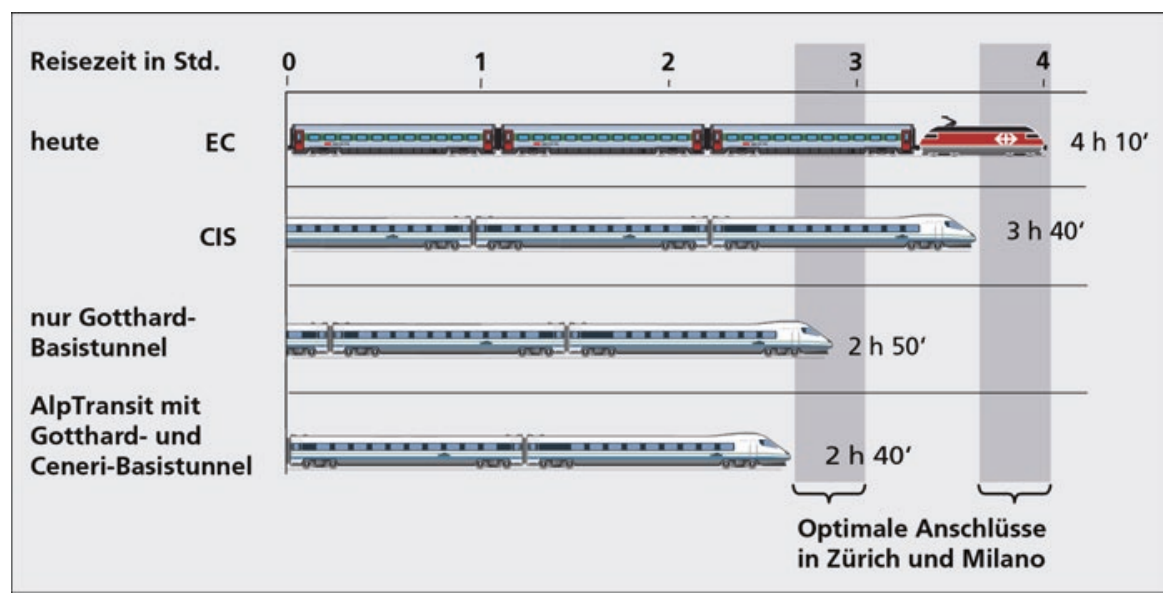

Abb. 11 Dank der NEAT geplante Fahrzeitverbesserungen Zürich - Mailand im Personenverkehr (Bild AlpTransit Gotthard AG)

Zugang zum europäischen Hochgeschwindigkeitsnetz zu erhalten. Die NEAT versprach diesen Zugang für grosse Teile der Schweiz via Basel in Richtung Deutschland und Frankreich sowie in Richtung Italien über Chiasso und Domodossola zu schaffen. Mailand sollte nach dem Endausbau dank der Neubaustrecke von Arth Goldau nach Lugano von Zürich und Luzern aus in gut $2 \frac{1}{2}$ Stunden erreichbar sein (Abb. 11), Rom in knapp 51/2 Std. Die Fahrzeit Lausanne - Mailand sollte 23/4 Stunden betragen ${ }^{12}$.

Grosse Teile des Fernreiseverkehrs sollten dank der durch die NEAT ermöglichten attraktiven Fahrzeiten ohne Zwang von der Strasse oder aus der Luft auf die Schiene verlagert werden.

\subsection{Versprechungen gegenüber den direkt Betroffenen}

Den Verantwortlichen für die Konzeption der NEAT war von Anfang an klar, dass den Umweltauswirkungen des Projekts grösste Aufmerksamkeit geschenkt werden musste. Zwar war der grösste Teil der NEAT-Neubaustrecken als Tunnelstrecken geplant, was eine grundsätzlich günstige Voraussetzung war. Der prognostizierte Landverbrauch belief sich dadurch auf nur 97 ha, was in Relation zur anfangs der Neunziger-Jahre in der Schweiz jährlich überbauten Fläche von rund 2000 ha ein sehr geringes Ausmass war. ${ }^{10}$ Der Landverbrauch war somit kein grundsätzliches Problem, allerdings wurde hoher Wert daraufgelegt, die an sich geringen Eingriffe

\footnotetext{
${ }^{10}$ Schweizerische Eidgenossenschaft, Parlamentsdienste, Verhandlungsheft 90.040, Neue Eisenbahn-Alpentransversale (NEAT), 1991.
} 
in die Natur mit entsprechenden Ersatzmaßnahmen zu kompensieren. Lokal war das Thema Umwelteingriffe aber von hoher Brisanz (vgl. Abschn. 3.5).

Die negativen Auswirkungen der neuen Eisenbahnlinie sollten durch eine sorgfältige Wahl der Linienführung und mit baulichen Massnahmen (z. B. für Lärmschutz, Umweltersatzmassnahmen) auf ein Minimum gesenkt werden. Gemäss Alpentransit-Beschluss waren auch die Zulaufstrecken lärmtechnisch zu sanieren. Bei allen Festlegungen von Massnahmen waren die Kantone aktiv in die Entscheidungen miteinbezogen, um möglichst umweltschonende Lösungen mit hoher Akzeptanz zu finden.

Den betroffenen Bergregionen wurde in Aussicht gestellt, dass der Bau der NEAT Arbeit und Verdienst schaffen würde. ${ }^{11}$

\subsection{Versprechungen gegenüber der Europäischen Gemeinschaft (heutige EU)}

Der Beschluss des Parlaments vom 4. Oktober 1991 über den Bau der NEAT war eine zwingende Voraussetzung für den erfolgreichen Abschluss der Transitverhandlungen der Schweiz mit der EG. Die Forderung nach einem Korridor für 40-Tonnen-Lastwagen wurde damals von der EG fallengelassen. Die NEAT war der Tatbeweis der Schweiz, dass man gewillt war, den Transportverpflichtungen gegenüber der Europäischen Gemeinschaft (EG) nachzukommen, aber nur auf Basis einer umweltverträglichen Lösung per Bahn. Der Vorrang des kombinierten Verkehrs Schiene/Strasse war im Rahmen der Verhandlungen unbestritten; weshalb die damals noch geltende 28-Tonnen-Limite der Schweiz von der EG noch anerkannt wurde. Mit dem Transitvertrag gewährleistete die EG im Gegenzug dem schweizerischen Transportgewerbe den Zugang zum EG-Binnenmarkt.

Mit dem Transitabkommen vom 2. Mai 1992 verpflichtete sich die Schweiz das Netz der SBB durch eine neue Linie von Arth-Goldau bis Lugano mit einem Basistunnel zwischen den Räumen Erstfeld/Silenen und Bodio und dasjenige der Berner Alpenbahn-Gesellschaft Bern-Lötschberg-Simplon (heutige BLS AG, Nachfolgeorganisation der Bern-Lötschberg-Simplon-Bahn) durch einen neuen Basistunnel zu erweitern. ${ }^{12}$

Mit diesen Massnahmen wurde in Aussicht gestellt, dass sich der Nord-SüdVerkehr durch die Schweiz dank diesen baulichen Massnahmen auf 67 Millionen Tonnen erhöhen wird, was damals rund der Hälfte des gesamten, für die Jahre 2010/2015 prognostizierten alpenquerenden Transportvolumens (Frankreich, Öster-

\footnotetext{
${ }^{11}$ Schweizerische Eidgenossenschaft, Volksabstimmung vom 27. September 1992, Erläuterungen des Bundesrates.

${ }^{12}$ Schweizerischer Bundesrat, Botschaft zum Transitabkommen zwischen der Europäischen Gemeinschaft und der Schweiz, sowie zur trilateralen Vereinbarung zwischen der Bundesrepublik Deutschland, der Schweiz und Italien über den kombinierten Verkehr Schiene/Strasse vom 13. Mai 1992.
} 
reich, Schweiz) entsprach. Allein beim kombinierten Verkehr sollte der vorgesehene Ausbau die Durchfahrt von täglich 260 Zügen mit 43 Millionen Tonnen Ladung pro Jahr ermöglichen ${ }^{15}$.

Die Durchfahrtszeit für einen Güterzug durch die Schweiz sollte sich von 1992 noch fünf Stunden auf etwas mehr als drei Stunden, nach Inbetriebnahme der NEAT verringern ${ }^{15}$.

\subsection{Versprechungen gegenüber den Automobilisten}

Die geplante Autobahnverbindung zwischen den Kantonen Bern und Wallis (Rawiltunnel) musste zu Beginn der Achtziger-Jahre aus geologischen Gründen fallen gelassen werden. Als Ersatz für die aus dem Nationalstrassennetz gestrichene RawilAutobahn sollte ein leistungsfähiger Autoverlad am Lötschberg-Basistunnel geschaffen werden. Autozüge mit Verladestationen in Heustrich bei Spiez auf der Nordseite und bei Steg in Rhonetal auf der Südseite sollten die Autobahnen N6 und N9 miteinander verbinden. Für das Kandertal sollte der neue Tunnel eine Entlastung vom Strassenverkehr ins Wallis und damit eine wesentlich umweltgerechtere Lösung bringen.

Zudem stellte man den Automobilisten generell in Aussicht, dass es dank dem kombinierten Güterverkehr im Nord-Süd-Transit und umgekehrt eine allgemeine Entlastung der Strassen geben werde, was zu weniger Staus führen sollte.

\subsection{Kosten und Termine/Versprechungen gegenüber dem Steuerzahler}

Das Parlament debattierte auf Basis der Botschaft vom 23. Mai 1990 über die NEAT. Der Projektstand 1988 mit Gesamtkosten von 10,1 Milliarden Schweizer Franken $(\mathrm{CHF})+40 \% /-10 \%$ (Preisbasis 1988) (Abb. 12) wurde auf den Projektund Preisstand 1991 hochgerechnet. Das Parlament genehmigte am 4. Oktober 1991 in Kenntnis der früher genannten Kostenbandbreiten einen Gesamtkredit von 14,0 Milliarden CHF für die Gotthard- und die Lötschberg-Achse sowie die Planungen für den Anschluss der Ostschweiz. Für die Bauausführung des Anschlusses Ostschweiz wurden weitere 850 Million CHF genehmigt. ${ }^{13}$

Dem Steuerzahler wurde in Aussicht gestellt, dass er als Mitglied der Erstellergeneration vom Tragen der finanziellen Last weitgehend verschont werde. $3 / 4$ der Mittel sollten den Bahnen über vom Bund am Kapitalmarkt beschaffte verzinsliche Darlehen zur Verfügung gestellt werden. Nur 1/4 der Investitionen waren vom Steuerzahler in Form von à fonds perdu-Beiträgen aufzubringen. Den Bahnen als künfti-

\footnotetext{
${ }^{13}$ Schweizerische Eidgenossenschaft, Parlamentsdienste, Verhandlungsheft 90.040, Neue Eisenbahn-Alpentransversale (NEAT), 1991.
} 


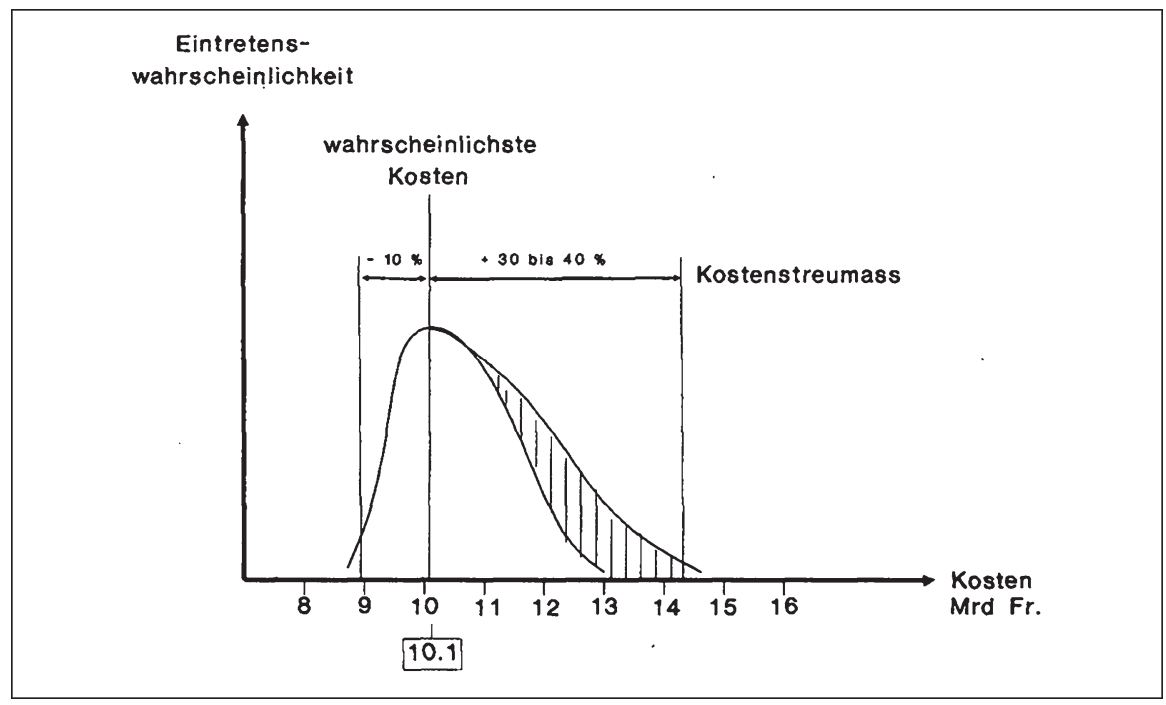

Abb. 12 Prognostizierte Gesamtkosten 1991 inkl. Prognosegenauigkeit für das NEAT Projekt, Projekt- und Preisstand 1988 (Bild Schweizerische Eidgenossenschaft (Schweizerischer Bundesrat, Botschaft über den Bau der schweizerischen Eisenbahn-Alpentransversale (AlpentransitBeschluss) vom 23. Mai 1990.))

gen Betreiberinnen der Infrastruktur und damit den künftigen Nutzern wurde die Pflicht auferlegt, die Darlehen nach der Inbetriebnahme innerhalb von 60 Jahren zurückzuzahlen. Aus betriebswirtschaftlicher Optik rechnete man mit einer Kostendeckung jedoch erst nach einer sehr langen Betriebszeit von 60-70 Jahren. ${ }^{14}$

Die benötigten Steuermittel in der Höhe von einem Viertel der Investitionskosten wären durch Treibstoffzollmittel zu decken gewesen, womit die positiven Effekte der NEAT zu Gunsten einer Entlastung der Strassen berücksichtigt werden sollten.

Allgemein war man in der politischen Entscheidungsfindung davon überzeugt, dass mit dieser Finanzierung die längerfristige Rentabilität der NEAT zu gewährleisten war und die NEAT auch wirtschaftlich interessant wäre.

\subsection{Versprechungen gegenüber den Betreibern}

Gemäss der Botschaft an das Parlament ${ }^{18}$ ging man pro Tag am Gotthard von 300 Güterzügen und am Lötschberg von 104 Güterzügen und 132 Autozügen aus. Dazu kommen noch Personenzüge mit einer stündlichen Verbindung am Lötschberg und zwei stündlichen Verbindungen am Gotthard (je eine in Richtung Zürich und Basel/ Luzern).

\footnotetext{
${ }^{14}$ Schweizerischer Bundesrat, Botschaft über den Bau der schweizerischen Eisenbahn-Alpentransversale (Alpentransit-Beschluss) vom 23. Mai 1990.
} 
Die neue Gotthard-Bahn sollte als Flachbahn ausgebildet werden (Abb. 13), d. h. sie sollte die Alpen mit maximalen Steigungen von weniger als $1 \%$ durchqueren. Mit geringerer Traktionsleistung sollten längere und schwerere Güterzüge möglich werden. Die Produktivität der Bahn sollte damit sowohl im Güter- als auch im Personenverkehr, auch dank der Streckenverkürzung und höheren Geschwindigkeiten erheblich gesteigert werden.

\section{Wurden oder werden die Versprechungen eingehalten?}

Zur Beantwortung dieser generellen Fragestellung müssen die folgenden Teilfragen beantwortet werden:

1. Wurde die Infrastruktur im versprochenen Umfang realisiert?

2. Kann der ursprünglich geplante Betrieb mit der gebauten Infrastruktur gewährleistet werden?

3. Welcher Betrieb wird tatsächlich gefahren?

4. Wurden Termine, Kosten und die Versprechungen an das Umfeld eingehalten?

\subsection{Gebaute Anlagen}

Nach der Volksabstimmung im September 1992 änderten sich die Rahmenbedingungen für das Projekt rasch. Zum einen lehnten die Schweizer im Dezember 1992 den Beitritt zum europäischen Wirtschaftsraum EWR ab, was zur Folge hatte, dass die EU den Druck auf die Schweiz erhöhte, insbesondere auch in Bezug auf Abschaffung der Limite von 28-Tonnen für das Gesamtgewicht von Lastkraftwagen. In einer politischen Gegenreaktion wurde 1994 vom Schweizervolk aufgrund einer Volksinitiative (Alpeninitiative) dem Verbot des Ausbaus der Strassenkapazitäten für den Alpentransitverkehr auf Verfassungsstufe zugestimmt. Die alpenquerenden Lastwagenfahrten sollten durch die Forderung, wonach der Transitverkehr von Grenze zur Grenze auf die Schiene zu verlagern wäre, innert 10 Jahren, d. h. bis 2014, auf ein Niveau von jährlich 650.000 Fahrten herunter gebracht werden. ${ }^{15}$ Diese Fakten erhöhten den Druck zum Bau der NEAT enorm.

Parallel dazu begannen sich aber die wirtschaftlichen Aussichten einzutrüben. Die Frage ob sich die Schweiz die NEAT im geplanten Ausmass finanziell leisten könne, wurde nochmals auf den Prüfstand gestellt, was zu intensive Diskussionen auf der Ebene des Bundesrates führte. Ein Gutachten eines vom Projekt vollkommen unabhängigen englischen Beratungsbüro, Coopers \& Lybrand, sollte helfen die Frage der Machbarkeit der vorgesehenen Finanzierung der NEAT zu klären. Coo-

\footnotetext{
${ }^{15}$ Schweizerische Eidgenossenschaft, Volksabstimmung vom 20. Februar 1994, Erläuterungen des Bundesrates.
} 


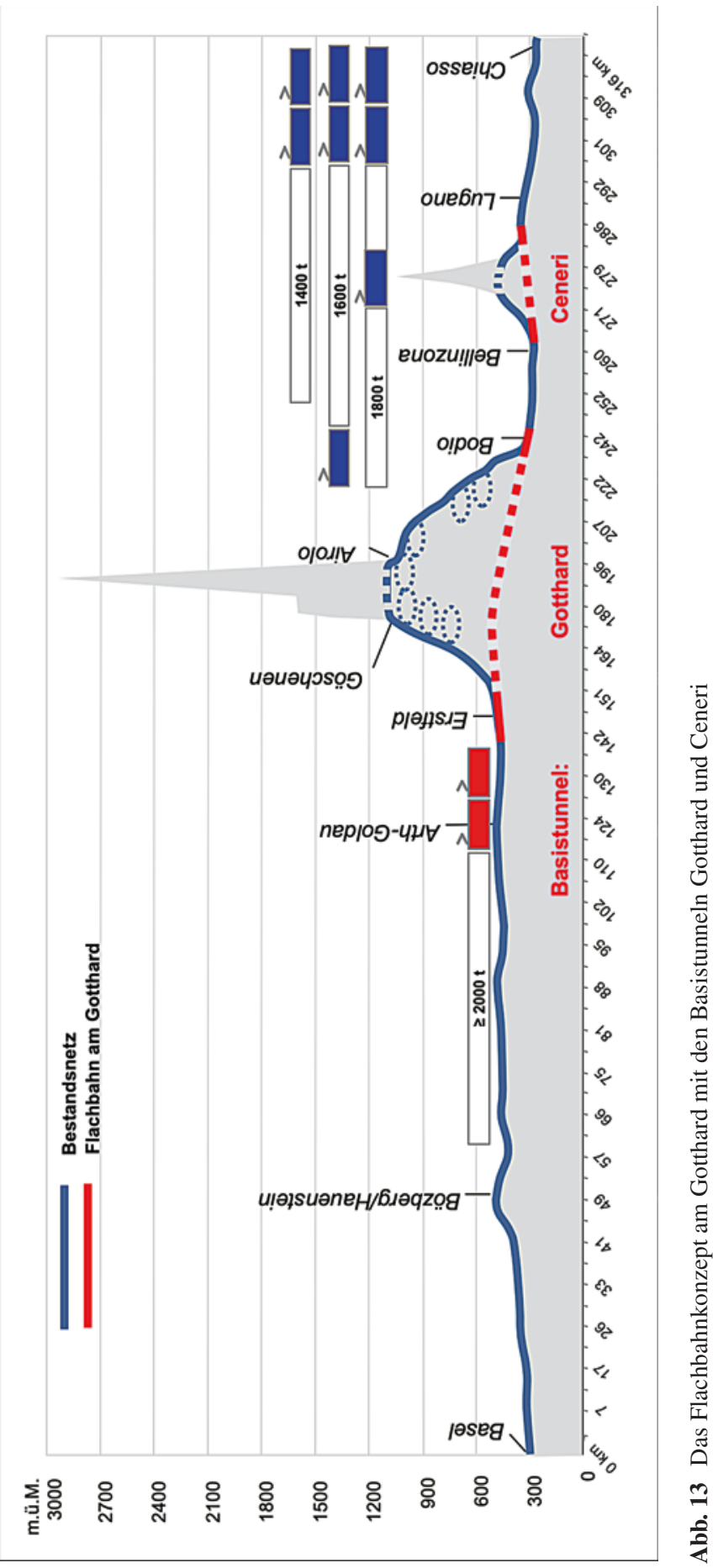


pers \& Lybrand kam zum Schluss, dass die NEAT bei der vorhandenen europäischen Tarifstruktur betriebswirtschaftlich nicht rentabel zu betreiben sein werde. Dies wäre nur dann der Fall, wenn für die teuren Neubaustrecken durch die Alpen ganz andere Tarife verlangt werden könnten. Solche Preisanpassungen wurden unter Berücksichtigung der europäischen Verkehrspolitik als äusserst unwahrscheinlich betrachtet. Die Bahngesellschaften als künftige Betreiberorganisationen wären unter diesen Randbedingungen nicht in der Lage gewesen, die Investitionen innert der angedachten 60 Jahre zurückzuzahlen und die entsprechenden Zinslasten zu tragen. ${ }^{16}$ Es mussten somit Lösungen gefunden werden.

Unter Berücksichtigung des genannten Gutachtens fällte der schweizerische Bundesrat nach einer Klausurtagung im Februar 1995 folgende Grundsatzentscheide. ${ }^{17}$

1. Die NEAT wird insgesamt als Netzvariante auf der Basis des Bundesbeschlusses vom Oktober 1991 realisiert: der Bau der beiden Basistunnel wird bestätigt.

2. Das Eidgenössische Finanzdepartement (EFD) erarbeitet, in Zusammenarbeit mit dem Eidgenössischen Verkehrs- und Energiewirtschaftsdepartement (EVED), Varianten für ein neues Finanzierungsmodell, das dem Bundesrat unterbreitet wird. Im Vordergrund steht eine zeitlich begrenzte und zweckgebundene Erhöhung des Treibstoffzolls zur Finanzierung der NEAT.

3. Das EVED erhält den Auftrag, in Zusammenarbeit mit dem EFD zu prüfen, wie die verschiedenen Baulose (v. a. Zufahrten) so organisiert werden können, dass ein möglichst gleichmässiger Finanzierungsbedarf über die Baujahre entsteht, welcher mit der vorgesehenen Finanzierung in Übereinstimmung gebracht werden kann.

4. Das EVED prüft zusammen mit den Kantonen kostengünstige Ausführungen betreffend Basis- und Alternativvarianten, um unter Berücksichtigung der Umweltverträglichkeit möglichst im ursprünglichen Rahmen der Kostenvoranschläge bleiben zu können.

5. Das EVED überprüft die Gestaltung des Autoverlades.

Diese Grundsatzentscheide wurden dann von 1996 bis 1998 im Rahmen der Ausarbeitung der Vorlage zum Bau und zur Finanzierung der Infrastruktur des öffentlichen Verkehrs (FinöV-Vorlage) wie folgt umgesetzt. ${ }^{18}$

Zu 2.) Der Anteil der von den Betreibern rückzahlbaren, am Kapitalmarkt aufgenommenen Darlehen wurde von $75 \%$ auf $25 \%$ gesenkt. Die zusätzlichen von der öffentlichen Hand aufzubringenden à fonds perdu Mittel sollten über zusätzliche neue Steuern (Anteile aus der Leistungsabhängigen Schwerverkehrsabgabe (LSVA), Anteile aus Mineralölsteuer, 0,1\%-Anteil der Mehrwertsteuer) finanziert werden. Die Finanzströme

\footnotetext{
${ }^{16}$ Coopers \& Lybrand, Financial review of the Neue Alpen-Transversale project, February 1995.

${ }^{17}$ Beschlüsse der Bundesratsklausur vom 19./20.2.1995.

${ }^{18}$ Schweizerischer Bundesrat, Botschaft über Bau und Finanzierung der Infrastruktur des öffentlichen Verkehrs vom 26. Juni 1996.
} 
sollten über einen Fonds zur Finanzierung der öffentlichen Verkehrsinfrastruktur geleitet (FinöV-Fonds) werden.

$\mathrm{Zu}$ 4.) Die NEAT sollte wie folgt redimensioniert und etappiert werden:

- Der Lötschberg-Basistunnel sollte nicht mehr zu $100 \%$, sondern nur noch zu rund $40 \%$ als vollwertiger Doppelspurtunnel ausgebaut werden, bei weiteren $40 \%$ war nur der Rohbau für die zweite Röhre zu erstellen und bei rund $20 \%$ der Gesamtlänge sollte der Tunnel einspurig mit einem nebenliegenden Sicherheitsstollen (Abb. 14) bleiben.

- Die Gotthardachse wurde etappiert. In einer ersten Etappe sollte der Gotthard-Basistunnel gebaut werden. Der Ceneri-Basistunnel, der zweite Bauabschnitt des Zimmerbergtunnels (Thalwil - Zug) und der Hirzeltunnel wurden in die zweite Etappe geschoben. Alle übrigen Bauten (Axentunnel, Verbindungsstrecke Gotthard - Ceneri mit der Umfahrung Bellinzona) wurden zurückgestellt (Abb. 15).

Zu 5.) Auf den Autoverlad am Lötschberg-Basistunnel wurde verzichtet. Es blieb beim bereits bestehenden Autoverlad am Lötschberg-Scheiteltunnel.

In Kenntnis dieser neuen Randbedingungen stimmten die Stimmbürger im Jahr 1998 sowohl der Einführung einer neuen Steuer in Form der Leistungsabhängigen

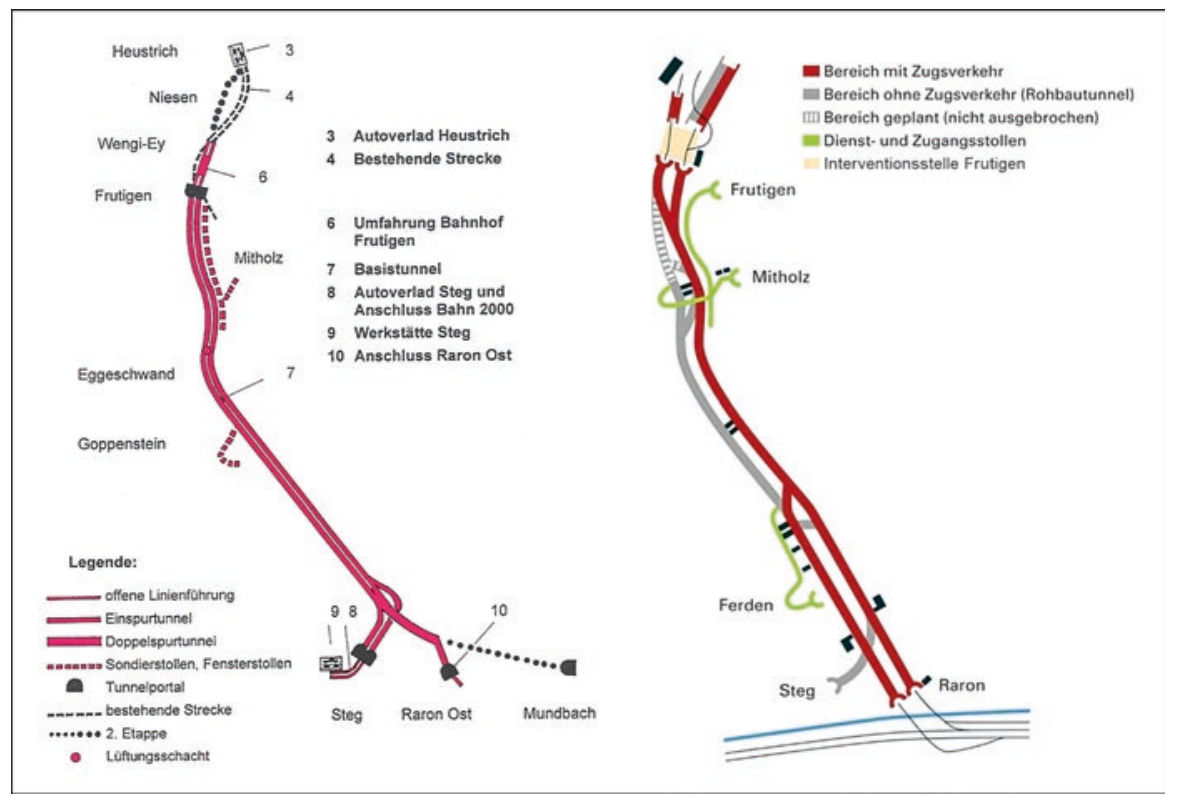

Abb. 14 Redimensionierung der NEAT 1998: aus dem durchgehend doppelspurigen LötschbergBasistunnel (links) wird ein mehrheitlich einspuriger Tunnel ohne Autoverlad (rechts) (Bilder BLS Alptransit AG) 

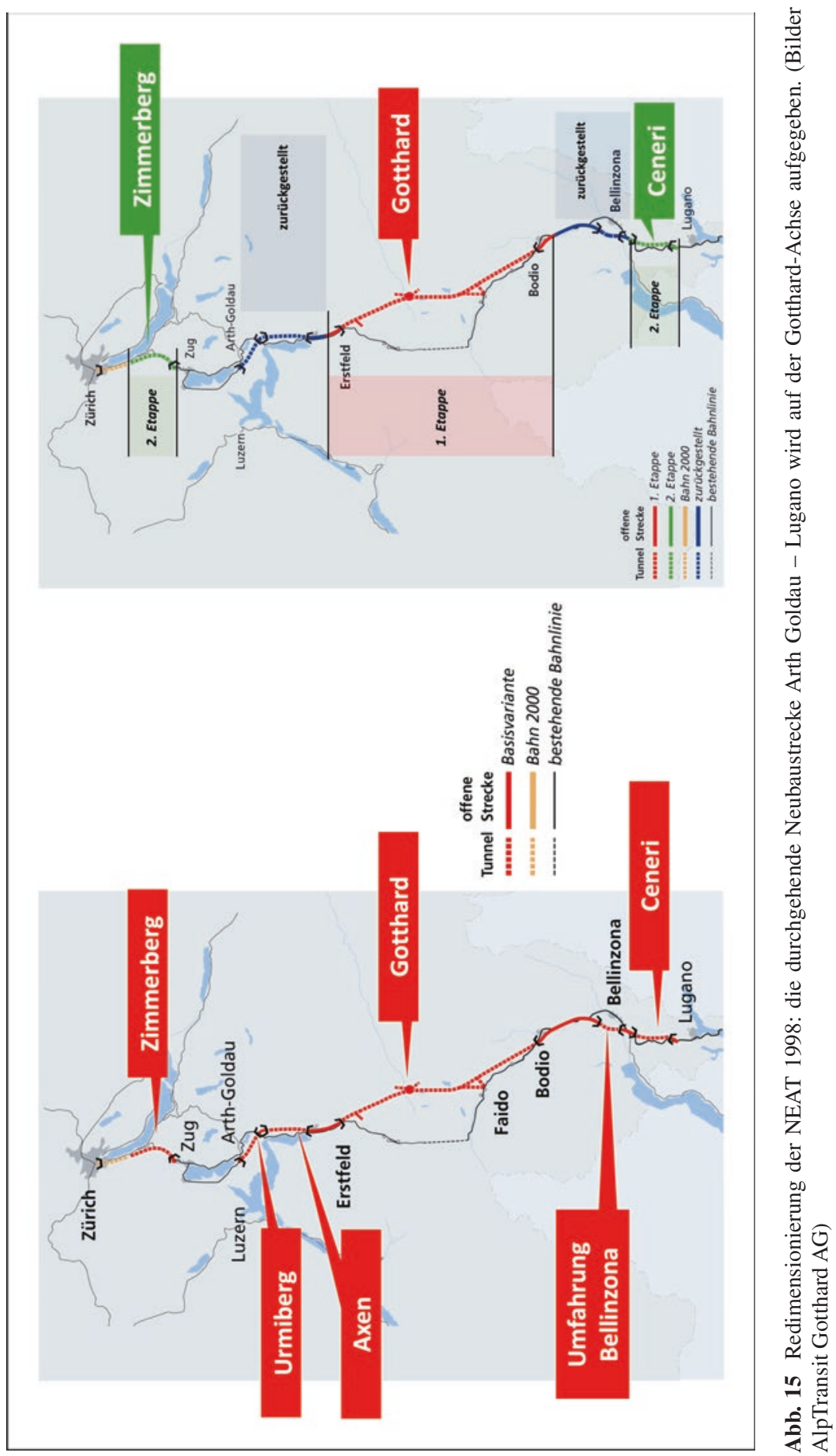
Schwerverkehrsabgabe (LSVA) als auch der Schaffung eines mehrheitlich mit Steuermitteln (Benzinzollzuschlag, Erhöhung der MwSt. und LSVA) geäufneten FinöV-Topfs, und damit implizit auch einer redimensionierten und etappierten NEAT zu (Abb. 14 und 15).

Mit diesen Beschlüssen konnten die Versprechen die Ostschweiz durch den Bau neuer Linien aus dem Raum Zug in den Raum Zürichsee besser anzuschliessen und am Lötschberg einen Autobahnersatz zu schaffen, nicht mehr eingehalten werden. Im Gegenzug wurden aber rund vier Milliarden CHF an Investitionskosten eingespart.

Im Jahr 2004 wurde dann der Ceneri-Basistunnel als einziges Objekt der zweiten Ausbauetappe vom Parlament zur Ausführung freigegeben (Abb. 16). Der zweite Bauabschnitt des Zimmerbergtunnels wurde 2008 definitiv aus dem NEAT-Bauprogramm gestrichen (Abb. 16), hat mittlerweile aber zusammen mit dem teilweisen Doppelspurausbau des Lötschberg-Basistunnels Eingang in das neue Finanzierungsprogramm zum Ausbau der Eisenbahninfrastruktur per 2035 gefunden. Durch diese zeitliche Etappierung entsteht ein erheblicher finanzieller Mehraufwand.

\section{Fazit 1: gebaute Infrastruktur}

Die durchgehende Neubaustrecke Arth Goldau - Lugano konnte aus wirtschaftlichen Gründen nicht realisiert werden. Auch auf die Anbindung der Ostschweiz via Hirzeltunnel musste verzichtet werden.

Am Lötschberg musste auf den durchgehenden Doppelspurtunnel verzichtet werden. Der Tunnel wurde zu rund $60 \%$ nur einspurig gebaut, was den Wegfall des Autoverlads als Ersatz für den Rawil-Autobahntunnel zur Folge hatte.

\subsection{Kann der ursprünglich geplante Betrieb gewährleistet werden?}

Es stellt sich nun die Frage, ob mit der reduzierten gebauten Infrastruktur gemäss FinöV-Konzept (1998) die übergeordneten Ziele der NEAT (1992) immer noch erreichbar waren.

Basis des FinöV-Konzepts 1998 war ein Fahrplan, welcher 370 Güterzüge durch die Schweizer Alpen vorsah (260 am Gotthard, 110 am Lötschberg). Damit war das übergeordnete Ziel der NEAT aus dem Jahr 2002, nämlich die Verlagerung der Güter auf die Schiene weiterhin erreichbar.

Auch die Zielsetzung von zwei stündlichen Personenzugsverbindungen pro Richtung durch den Gotthard-Basistunnel und einer stündlichen Verbindung durch den Lötschberg waren mit dem angepassten Konzept immer noch realisierbar. Der Verzicht auf die durchgehende Hochgeschwindigkeitsstrecke Arth Goldau - Lugano 


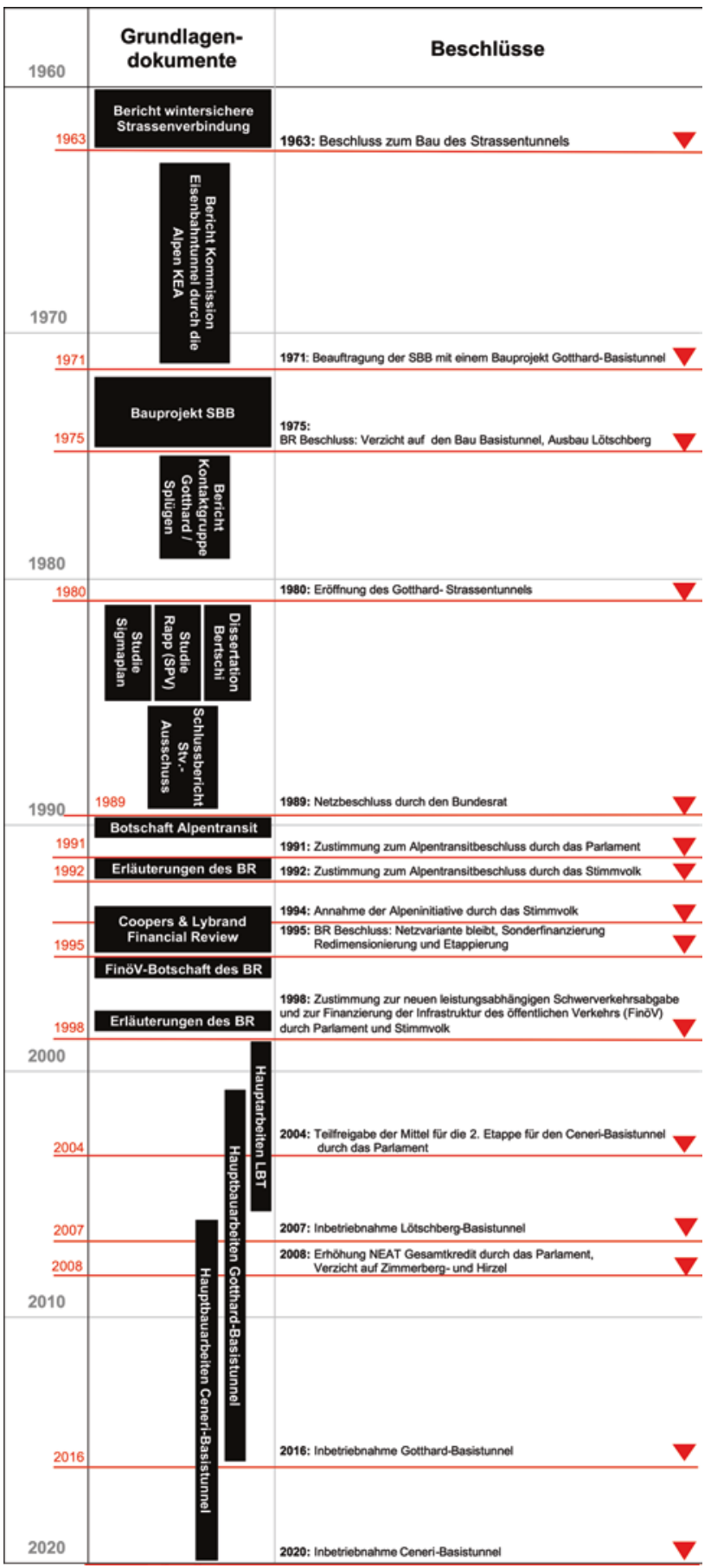

Abb. 16 Zeitschiene mit den wichtigsten Grundlagendokumenten und Entscheidungen zur NEAT 
hatte primär Auswirkungen auf die Fahrzeiten nicht aber auf die Kapazität des Systems.

Mit den fertig gestellten Transitachsen am Lötschberg und am Gotthard stellt die Schweiz nun eine Infrastruktur zu Verfügung, mit welcher die geplanten Zugzahlen von insgesamt 370 Güterzügen pro Tag gefahren werden können, womit eine Transitkapazität von 67 Mio. Tonnen zur Verfügung steht. Dies entspricht, wie versprochen, etwas mehr als der Hälfte des gesamten aktuellen Gütertransitverkehrs über die Alpen (2018: 117,4 Mio. Tonnen). Die im Rahmen der Verhandlungen zum Transitabkommen gegenüber der EU getätigten Versprechungen wurden damit eingehalten. $^{19}$

Auch die in Aussicht gestellten Personenverkehrstrassen können sowohl am Gotthard wie auch am Lötschberg gefahren werden.

\section{Fazit 2: Vorhandene Kapazitäten}

Trotz redimensionierter Infrastruktur können die versprochenen Kapazitäten für den Personen- und den Güterverkehr zur Verfügung gestellt werden.

\subsection{Bisher erbrachte Leistungen im Güterverkehr}

Es stellt sich somit die Frage, ob die vorhandenen Kapazitäten von den Eisenbahnverkehrsunternehmen (EVU) auch genutzt werden können und ob die angebotenen Verkehrsdienstleistungen die erwartete hohen Qualität aufweisen. Dabei kann es derzeit nicht darum gehen, ob die langfristig geschaffenen Kapazitäten schon voll ausgenutzt sind, sollen doch noch Reserven für die Zukunft erhalten bleiben. Vielmehr interessiert aktuell ob und wie sich der Modal-Split im transalpinen Güterverkehr entwickelt, ob es zu einem Trendbruch zu Gunsten der Schiene kommt und ob das aus der Annahme der Alpeninitiative längst überfällige Ziel von maximal 650.000 Lastwagenfahrten pro Jahr durch die Schweiz erreicht werden kann.

Im Herbst 2020 ist es für ein abschliessendes Urteil noch zu früh, insbesondere weil die Aufweitung der Zulaufstrecken am Gotthard auf 4 Meter Durchfahrthöhe (4 m-Korridor) erst 2020 in Betrieb gehen wird. Erste Indikatoren zum Stand der Dinge gibt es aber trotzdem.

Die schweizerische Eidgenossenschaft veröffentlicht alle zwei Jahre einen Bericht zum alpenquerenden Güterverkehr ${ }^{23}$. Aus dem im November 2019 publizierten Bericht lässt sich keine markante Zunahme des Güterverkehrs auf der Strasse und der Schiene feststellen. Der alpenquerende Güterverkehr durch die Schweiz stagniert seit dem Jahr 2008 bei einem Gesamtvolumen von ca. 40 Mio. Tonnen. Bei

\footnotetext{
${ }^{19}$ Schweizerischer Bundesrat, Botschaft zum Transitabkommen zwischen der Europäischen Gemeinschaft und der Schweiz, sowie zur trilateralen Vereinbarung zwischen der Bundesrepublik Deutschland, der Schweiz und Italien über den kombinierten Verkehr Schiene/Strasse vom 13. Mai 1992.
} 


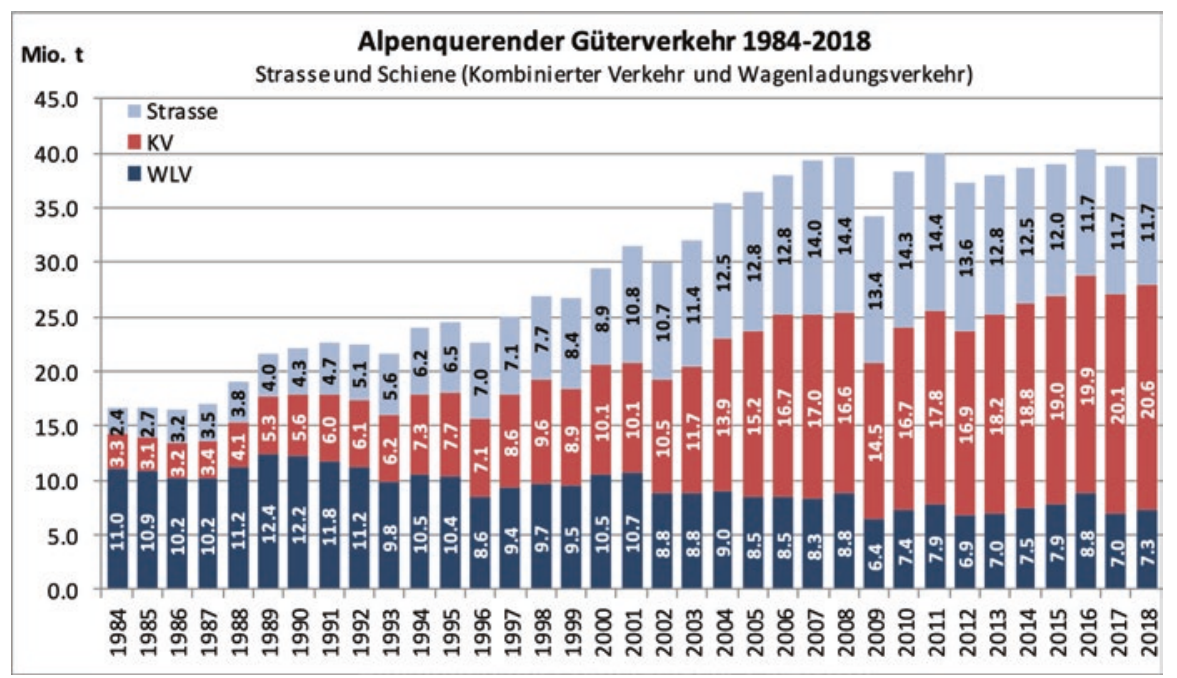

Abb. 17 Alpenquerender Güterverkehr durch die Schweiz 1984-2018 (Bildquelle Schweizerische Eidgenossenschaft, Bericht des Bundesrats, Bericht über die Verkehrsverlagerung vom November 2019, Verlagerungsbericht Juli 2017 - Juni 2019.)

der Mengenentwicklung ist weder im Jahr 2008 ff. ein „Lötschberg-Effekt“ noch im Jahr 2017 ff. ein „Gotthard-Effekt“ erkennbar (Abb. 17).

Der einzige positiv nachweisbare Effekt ist die Veränderung im Model-Split zwischen Schiene und Strasse von $64 \%$ zu $36 \%$ im Jahr 2007 zu $70 \%$ zu $30 \%$ im Jahr 2018 (Abb. 18). Die Bahn holt sich langsam, aber stetig Marktanteile zurück. 2018 erfolgten weiterhin 941.000 Fahrten mit schweren Güterfahrzeugen auf der Strasse durch die Schweizer Alpen (gegenüber 2.422.000 Fahrten am Brenner), was gegenüber dem Verlagerungsziel immer noch 291.000 Fahrten zu viel sind. In der Zeit zwischen 2011 und 2018 gingen die Lastwagenfahrten durch die Schweiz bei praktisch gleichbleibendem Transportvolumen um 279.000 Fahrten zurück. Bei unverändertem Trend wäre das Verlagerungsziel erst in 8 bis 10 Jahren und damit mit rund 15 Jahren Verspätung erreichbar.

Die Ursachen für das nicht steigende Gesamtgütervolumen durch die Schweiz dürften vielfältig sein. Zum einen spielt sicher die wirtschaftliche Entwicklung der Wirtschaftsräume entlang der Transitkorridore beidseits der Alpen eine wichtige Rolle. In den vergangenen Jahren waren in den osteuropäischen Ländern höhere Wachstumsraten zu verzeichnen als in Zentral- und Westeuropa. Dieses Faktum, zusammen mit der fehlenden Eisenbahninfrastruktur auf den Zulaufstrecken dürfte eine der Ursachen für eine gegenüber der Brennerachse andersartige Entwicklung auf den schweizerischen Transitachsen sein. ${ }^{20}$

\footnotetext{
${ }^{20}$ Schweizerische Eidgenossenschaft, Bericht des Bundesrats, Bericht über die Verkehrsverlagerung vom November 2019, Verlagerungsbericht Juli 2017 - Juni 2019.
} 


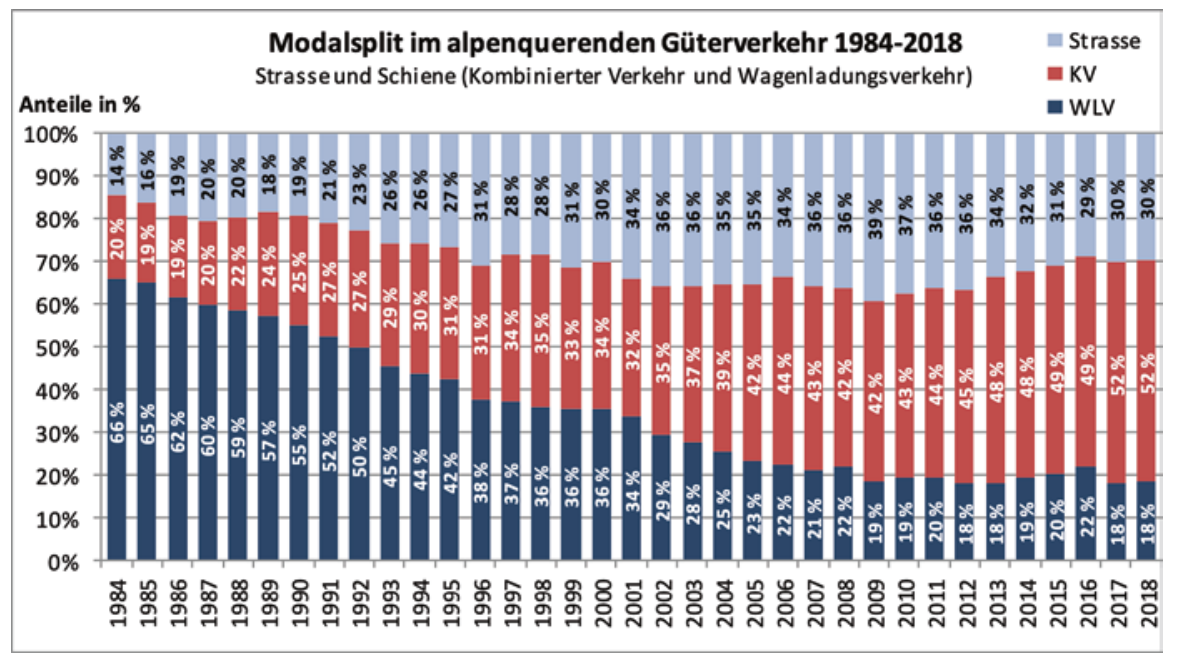

Abb. 18 Entwicklung des Modalsplit im alpenquerenden Güterverkehr durch die Schweiz seit 1984 (Bildquelle ${ }^{24}$ )

Die fehlenden Durchfahrtshöhen bei den Zulaufstrecken, fehlende Terminalkapazitäten, um die Container auch auf die Züge und von den Zügen auf die Strasse zu bringen sowie die Havarie beim Tunnel Rastatt am 12. August 2017 bei nicht vorhandenen Umleitstrecken dürften weitere Gründe für die genannte Entwicklung sein.

Wenn schon keine höheren Mengen transportiert wurden, so stellt sich die Frage, ob sich die Qualität der Transportdienstleistungen markant gesteigert hat. Dazu stellt der Verlagerungsbericht 2019 folgendes fest. ${ }^{21}$

\begin{abstract}
„Die Pünktlichkeit des alpenquerenden kombinierten Verkehrs hat sich im Berichtszeitraum (Juni 2017 - Juni 2019), verschlechtert: Im Mittel erreichte im 1. Semester 2019 weniger als die Hälfte (43\%) aller Züge ihr Ziel pünktlich (Verspätungen zwischen 0 und 30 Minuten). Auch der Anteil grosser Verspätungen (über 3 Stunden) nahm zu und lag im 1. Semester 2019 bei $29 \%$. Mehr als ein Viertel der Züge des kombinierten Verkehres erreichte im 1. Semester 2019 sein Ziel also mit einer Verspätung von mehr als drei Stunden. Für den Wagenladungsverkehr (WLV) ist von ähnlichen Pünktlichkeitswerten auszugehen.

Für die schlechte Qualität sind zahlreiche Ursachen verantwortlich. Infrastrukturseitig führen vor allem die seit langer Zeit bestehenden, intensiven Bauarbeiten auf dem Nord-Süd-Korridor (vor allem in Deutschland und Italien) zu zeitweiligen Kapazitätseinschränkungen (z. B. eingleisigem Betrieb) oder erfordern zeitweise Streckensperrungen mit Umleitungen. Hinzu kommen Ressourcenengpässe bei Lokführern und Traktion einiger Unternehmen. Auch externe, nicht beeinflussbare Ereignisse wie Streiks, witterungsbedingte Sperrungen oder Suizide haben grosse Auswirkungen. Im operativen Betrieb geniesst der Güterverkehr zudem keine Priorität, so dass er bei der Rückkehr zum Normalbetrieb oft hinter dem Personenverkehr anstehen muss. Verspätungen eines Zugs bei Ankunft am Terminal resultieren oft in Folgeverspätungen, da Zugskompositionen erst ver-
\end{abstract}

\footnotetext{
${ }^{21}$ Schweizerische Eidgenossenschaft, Bericht des Bundesrats, Bericht über die Verkehrsverlagerung vom November 2019, Verlagerungsbericht Juli 2017 - Juni 2019.
} 


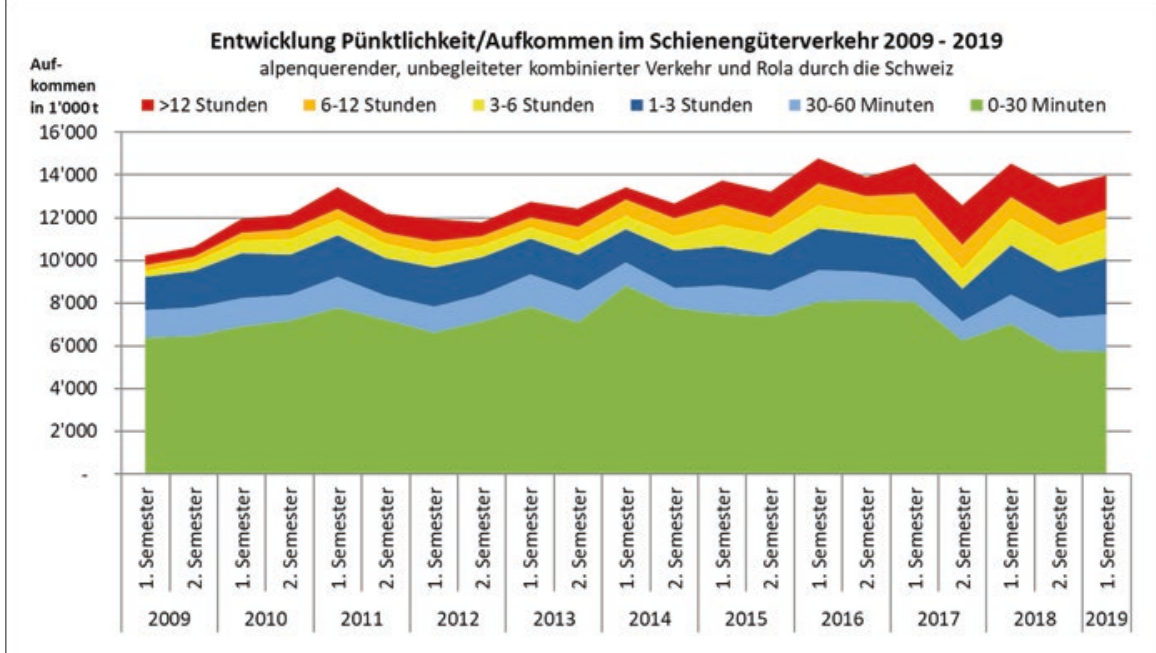

Abb. 19 Entwicklung Pünktlichkeit/Aufkommen im alpenquerenden Schienengüterverkehr durch die Schweiz 2009-2019 (Bildquelle (Schweizerische Eidgenossenschaft, Bericht des Bundesrats, Bericht über die Verkehrsverlagerung vom November 2019, Verlagerungsbericht Juli 2017 - Juni 2019.))

spätet für den Wiederbelad zur Verfügung stehen. ... Es zeigt sich, dass sich heute die Menge, welche pünktlich (d. h. mit höchstens 30 Minuten Verspätung) am Terminal ankommt, auf dem Niveau von 2009 bewegt. Mit dem Zuwachs an Aufkommen seit 2009 haben, absolut betrachtet, auch die grossen Verspätungen zugenommen."

Die Qualität der Gütertransportdienstleistungen hat sich seit 2009 somit verschlechtert (Abb. 19). Bei Industriezweigen, welche auf just-in-time-Produktion beruhen, ist die Verlässlichkeit der Transportdienstleistung aber von höchster Wichtigkeit. Die Infrastrukturbetreiber und die beteiligten Eisenbahnverkehrsunternehmungen sind derzeit trotz der getätigten enormen Investitionen in eine hochwertige Transitinfrastruktur in der Schweiz nicht in der Lage die erwartete Qualität zu liefern.

Die Ursachen liegen, wie im Verlagerungsbericht ${ }^{27}$ analysiert wird, primär im europaweiten Gesamtsystem Eisenbahn und nicht bei den schweizerischen Neubaustrecken, welche eine ausgesprochen hohe Verfügbarkeit aufweisen.

\section{Fazit 3: Güterverkehr}

Im Güterverkehr holt sich die Bahn dank verbesserter Infrastruktur zuvor verlorene Marktanteile, bei von 2007 bis 2018 mehr oder weniger konstantem Verkehrsvolumen, stetig zurück. Bei unverändertem Trend wird das Verlagerungsziel aus der Alpeninitiative in acht bis zehn Jahren erreicht werden.

Die derzeitige Pünktlichkeit im Güterverkehr über die Alpen ist aufgrund ungenügender Infrastruktur auf den Anschlusstrecken und in den Terminals unbefriedigend, d. h. primär aufgrund der trotz entsprechender Absichtserklärungen ungenügenden Koordination im europäischen Gesamtsystem. 


\subsection{Bisher erbrachte Leistungen im Personenverkehr}

Beim Personenverkehr ergibt sich gegenüber dem Güterverkehr ein komplett anderes Bild. Der Lötschberg-Basistunnel ging 2007 in Betrieb und hat sich insbesondere im Personenverkehr als der grosse Renner erwiesen. Mit einer Fahrzeit von 56 Minuten von Bern nach Visp oder von zwei Stunden von Zürich nach Visp ist das Wallis viel besser erreichbar geworden. Die 1992 prognostizierten Fahrzeiten werden fast zu $100 \%$ erreicht. Damit ist es möglich geworden im Wallis zu wohnen und in Bern zu arbeiten oder einen Tagesausflug von Zürich z. B. nach Zermatt zu machen. Auch vom Zürcher Flughafen aus ist das Wallis viel besser erreichbar.

Auch am Gotthard gab es für den Personenverkehr rasch erste Erfolge. Die Zahl der Passagiere konnte von täglich 9000 auf aktuell 13.000 Passagiere gesteigert werden, was einen spürbaren Effekt im Modal-Split zwischen Strasse und Schiene auf der Gotthardroute darstellt. Der Erfolg war so groß, dass es an Feiertagen und Wochenenden in Einzelfällen zu Engpässen und überfüllten Zügen kam, so dass Passagiere zum Aussteigen aufgefordert werden mussten, um eine sichere Durchfahrt durch den Basistunnel zu gewährleisten.

Bezüglich des Einhaltens der Fahrzeiten ergibt sich jedoch sowohl bezüglich der aktuellen Situation als auch für die künftigen Prognosen ein weniger klares Bild (Tab. 1).

Bei der Abstimmung im September 1992 wurden dem Stimmbürger sehr kurze Fahrzeiten, u. a. die Halbierung der Fahrzeiten zwischen Luzern/Zürich und Bellinzona als Fernziel genannt. Aufgrund geänderter wirtschaftlicher Randbedingungen musste das NEAT-System im Jahr 1998 redimensioniert werden, womit rund 4 Milliarden Investitionsvolumen eingespart wurden. Mit dem FinöV-Konzept 1998 wurden die Zulaufstrecken mit dem Urmibergtunnel, dem Axentunnel, an der Tessiner Riviera und die Umfahrung Bellinzona aus dem Projektperimeter entfernt (Abb. 15) und im Hinblick auf künftige Ausbauprogramme zurückgestellt. Damit wurde auf der Gotthardachse auf den Bau einer durchgehenden Hochgeschwindigkeitsstrecke

Tab. 1 Fahrzeiten unter Berücksichtigung des NEAT-Ausbaus

\begin{tabular}{|c|c|c|c|c|c|}
\hline von & nach & $\begin{array}{l}\text { Abstimmungserläuterungen } \\
1992^{\mathrm{a}}\end{array}$ & $\begin{array}{l}\text { FinöV- } \\
\text { Botschaft } \\
1996^{\mathrm{b}}\end{array}$ & $\begin{array}{l}\text { Prognose } \\
2021\end{array}$ & $\begin{array}{l}\text { Erfüllungsgrad } \\
\text { gegenüber } \\
\text { 1992/FinöV }\end{array}$ \\
\hline Zürich & Bellinzona & $60 \mathrm{~min}$ & $95 \mathrm{~min}$ & $105 \mathrm{~min}$ & $90,5 \%$ \\
\hline Zürich & Lugano & $75 \min$ & $110 \mathrm{~min}$ & $120 \min$ & $91,7 \%$ \\
\hline Zürich & Mailand & $150 \mathrm{~min}$ & $165 \mathrm{~min}$ & $182 \mathrm{~min}$ & $90,7 \%$ \\
\hline Bern & Brig & $60 \mathrm{~min}$ & - & $65 \mathrm{~min}$ & $92,3 \%$ \\
\hline Bern & Stresa & $120 \mathrm{~min}$ & - & $125 \mathrm{~min}$ & $96,0 \%$ \\
\hline Bern & Mailand & & $165 \mathrm{~min}$ & $186 \min$ & $88,7 \%$ \\
\hline Neuenburg & Visp & $105 \mathrm{~min}$ & - & $118 \mathrm{~min}$ & $89,0 \%$ \\
\hline
\end{tabular}

${ }^{a}$ Schweizerische Eidgenossenschaft, Volksabstimmung vom 27. September 1992, Erläuterungen des Bundesrates

${ }^{\mathrm{b} S c h w e i z e r i s c h e r ~ B u n d e s r a t, ~ B o t s c h a f t ~ u ̈ b e r ~ B a u ~ u n d ~ F i n a n z i e r u n g ~ d e r ~ I n f r a s t r u k t u r ~ d e s ~ o ̈ f f e n t l i-~}$ chen Verkehrs vom 26. Juni 1996 
verzichtet, was zu entsprechenden Fahrzeitverlängerungen im Personenverkehr führte.

Mit der Botschaft über den Bau und die Finanzierung der Infrastruktur des öffentlichen Verkehr vom 26. Juni 1996 an das schweizerische Parlament, wurden den Entscheidungsträgern die neuen, verlängerten Fahrzeiten dargelegt, welche somit den Referenzpunkt für die möglichen Fahrzeiten nach der Eröffnung des CeneriBasistunnels bilden.

Wie ein Blick auf Tab. 1 zeigt werden die 1998 in Aussicht gestellten Fahrzeiten zu rund $90 \%$ erreicht. Die Gründe für die Differenz zu $100 \%$ liegen auf der Gotthard-Achse zum einen darin, dass im Jahr 2008 definitiv auf den zweiten Bauabschnitt des Zimmerberg-Basistunnels verzichtet wurde. Zum andern kommt es mittlerweile aufgrund anderer betrieblicher Abläufe als ursprünglich in Italien zu längeren Fahrzeiten auf den Anschlusstrecken.

\section{Fazit 4: Personenverkehr}

Im Gegensatz zum Güterverkehr ist im Personenverkehr eine starke Zunahme in der Nachfrage an Transportdienstleistungen festzustellen. Diese kann (abgesehen von wenigen Spitzentagen) zur Zufriedenheit der Kunden erbracht werden.

Die mit dem FinöV-Fahrplan in Aussicht gestellten Fahrzeiten können aufgrund des Verzichts auf die zweite Etappe des Zimmerberg-Basistunnels und aus betrieblichen Randbedingungen noch nicht vollständig erfüllt werden.

\subsection{Einhalten der Versprechungen gegenüber verschiedenen Interessenspartnern}

Das Versprechen gegenüber dem heutigen Steuerzahler, ihn mehrheitlich vom Tragen der finanziellen Lasten zu befreien, musste aus betriebswirtschaftlichen Überlegungen in zwei Schritten (1998 und 2005) fallen gelassen werden. Die Betreiberorganisationen wären auch innert 60 Jahren nicht in der Lage gewesen die Darlehen samt Zinsen zurückzuzahlen. Die finanziellen Lasten wurden mit dem FinöVFinanzierungsmodell (1998) und der Gesamtschau zur NEAT-Kostenentwicklung $(2005)^{22}$ vollständig vom künftigen Nutzer auf den heutigen Steuerzahler transferiert (Abb. 20). Dieser hat diesem Transfer mit den Volksabstimmungen zur LSVA und zum FinöV-Fonds im Jahr 1998 jedoch zugestimmt.

Der Landverbrauch für den Bau der NEAT war wie prognostiziert gering. Trotzdem wurde im Kanton Uri in jahrelangen Verfahren um die optimale Linienführung und die Dimensionierung der Anlagen auf dem knappen flachen Talboden gerungen. Konfliktpotenziale waren der Lärmschutz, der Hochwasserschutz und allfällige

\footnotetext{
${ }^{22}$ Schweizerischer Bundesrat, Botschaft zur Gesamtschau FinöV vom 17. Oktober 2007.
} 


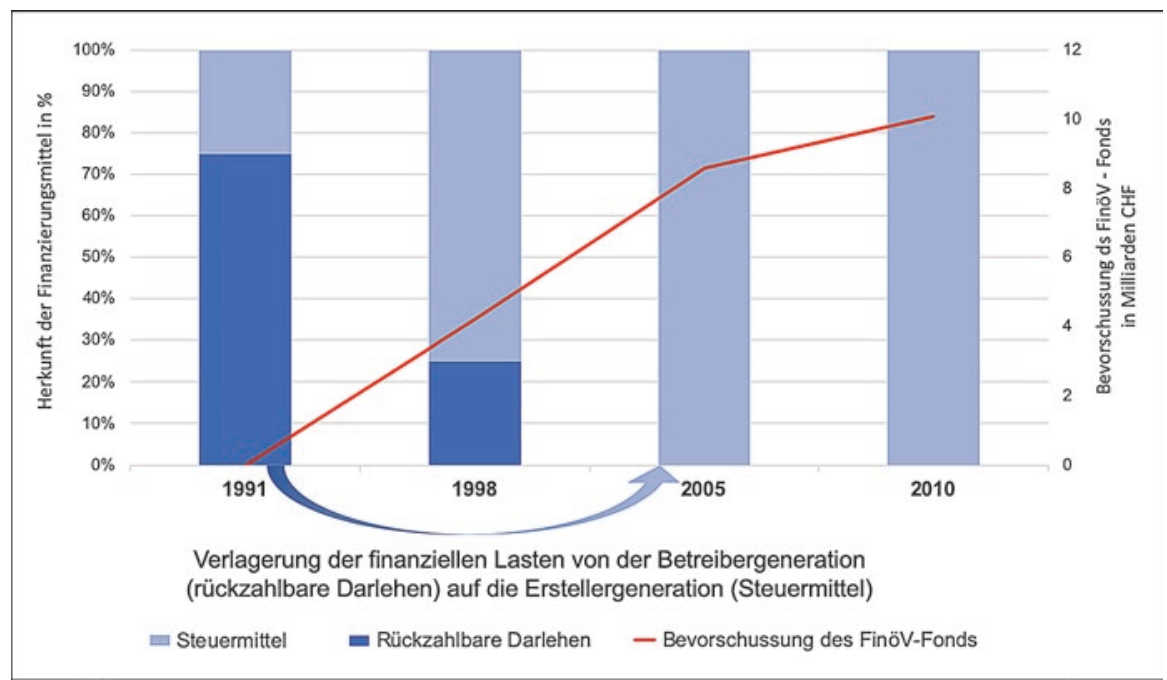

Abb. 20 Entwicklung der NEAT-Finanzierung, Umlagerung vom künftigen Nutzer auf den heutigen Steuerzahler (eigene Darstellung)

Störfälle. Schliesslich wurde in einem langwierigen Einigungsverfahren ein einvernehmlicher Kompromiss für die offene Strecke im Norden gefunden.

Das Gesamtterminprogramm erlitt dadurch aber mindestens ein Jahr Verzögerung. ${ }^{23}$

Im Teilabschnitt Sedrun konnten mit einer Langzeitstudie der Universität St. Gallen $^{24}$ die Auswirkungen der NEAT-Baustelle auf das Zusammenleben mit der Bevölkerung beobachtet und dokumentiert werden. Die Studie zeigt, dass die getätigten Versprechungen bezüglich der positiven wirtschaftlichen Auswirkungen und dem Ernstnehmen der Anliegen gegenüber der Bevölkerung und der Umwelt eingehalten wurden. Dank der Quellensteuereinnahmen kam die Gemeinde zu erheblichen zusätzlichen Steuereinnahmen, nebst dem, dass das lokale Gewerbe und das Gastgewerbe aus der Baustelle die versprochene Beschäftigung und entsprechenden Profit erhielten. Die Baustelle wurde von der großen Mehrheit der Bevölkerung nicht als störend empfunden (Abb. 21).

Die Redimensionierung der NEAT im Jahr 1998 ging zu Lasten der Autofahrer für Fahrten aus dem Kanton Bern ins Wallis und umgekehrt. Um die Personen- und Güterzugskapazitäten am Lötschberg zur Verfügung stellen zu können, musste der Autoverlad durch den Basistunnel als Autobahnersatz aufgegeben werden. Der Autoverlad steht weiterhin nur am Lötschberg-Scheiteltunnel zur Verfügung, womit

\footnotetext{
${ }^{23}$ Zbinden, Peter, Ehrbar, Heinz; Das Bewilligungsverfahren oder: Vom Wunsch zur Wirklichkeit; Tunnelling the Gotthard, Fachgruppe für Untertagbau, 2016.

${ }^{24}$ Universität St. Gallen, NEAT Begleitforschung Langzeitstudie Sedrun, Schlussbericht, St. Gallen 2016.
} 


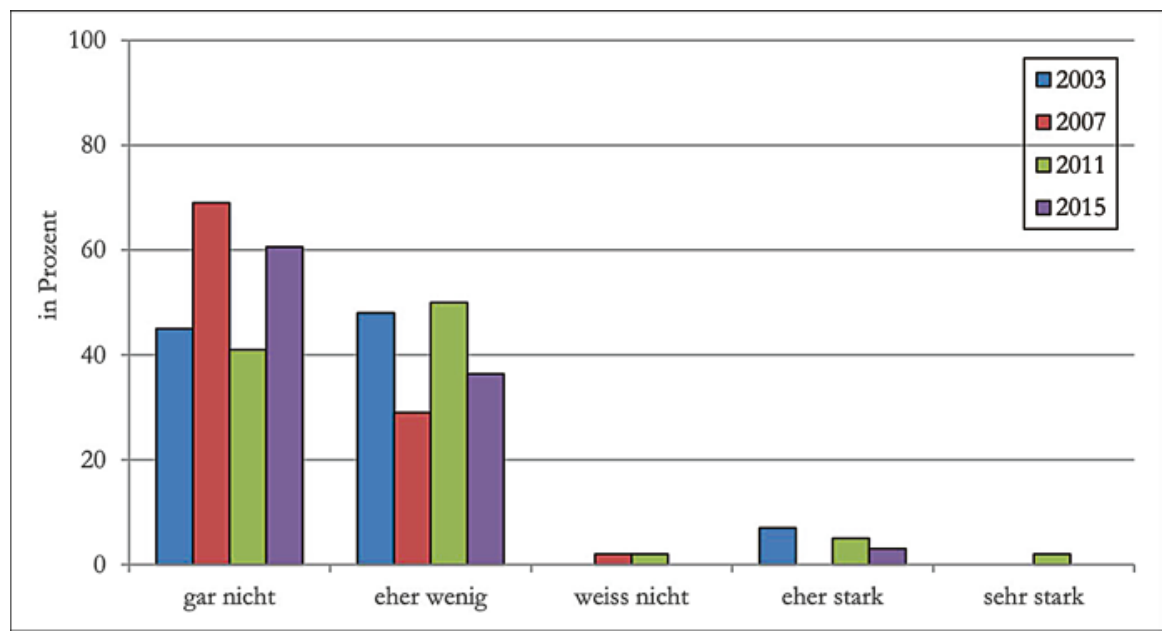

Abb. 21 Von der örtlichen Bevölkerung in Sedrun wahrgenommene Belästigung (Bildquelle28)

die Autos weiterhin durch das Kandertal hochfahren müssen. Das Versprechen des Autobahnersatzes konnte nicht eingelöst werden.

Gegenüber der EU und den Nachbarländern wurden die Versprechungen bezüglich der zur Verfügung gestellten Transportkapazitäten jedoch vollumfänglich eingehalten.

$\mathrm{Zu}$ guter Letzt wurde den Unternehmern versprochen, dass die Investitionen der Wirtschaft in Form von Beschäftigung, Gewinn und Reputationsgewinn sorgen sollten. Die enormen Investition haben während langen Jahren zu Spitzenzeiten für über 2500 Personen Beschäftigung gebracht. Von all den Beschäftigten stammten über $80 \%$ aus der Schweiz und den Nachbarländern. Hat sich auch der wirtschaftliche Erfolg für die betroffenen Unternehmer eingestellt?

Am Swiss Tunnel Congress 2013 in Genf zog ein Unternehmervertreter die folgende Bilanz: ${ }^{25}$

- Finanziell

- bezüglich Erfahrung und Know-how

- für die Reputation

- für die Referenzen

- für die Persönlichkeitsbildung

- für die Partnerinteressen nur in einem von fünf Hauptlosen lohnend

lohnend

lohnend

lohnend

lohnend

lohnend

${ }^{25}$ Gruber, Luzi R.; Gotthard-Basistunnel - Lohnt sich ein Werkvertrag für ein Megabaulos? Tagungsband Swiss Tunnel Congress 2013, Fachgruppe für Untertagbau, 2013. 


\subsection{Einhalten von Terminen und Kosten}

In den frühen Phasen wurden die Termin- und Kostenziele mit den entsprechenden Bandbreiten kommuniziert. Am Gotthard ging man von einer Bauzeit von 12 bis 15 Jahren aus. Nach den mehrjährigen Vorarbeiten starteten die eigentlichen Hauptarbeiten im Jahr 2001. Im Mai 2016 wurde der Gotthard-Basistunnel für die Inbetriebnahme übergeben. Die 15 Jahre Bauzeit sind eingehalten worden. Desgleichen ging der Lötschberg-Basistunnel, wie vereinbart, auf den Tag genau im Dezember 2007 in Betrieb.

Im Zuge der Redimensionierung der NEAT im Jahre 1998 wurden auch die Kosten neu definiert. Aus dem vom Parlament für die gesamte NEAT im Jahr 1991 genehmigten Gesamtkredit von 14,9 Milliarden CHF (Preisstand 1991) wurden nach der Projektredimensionierung im Jahr 1998 dann 12,2 Milliarden CHF (Preisstand 1998) als ursprüngliche Kostenbezugsbasis (UKB) für die künftige finanzielle Steuerung festgelegt. Die aktuelle Kostenprognose der Erstellergesellschaften rechnet mit abschliessenden Gesamtkosten von 17.695 Milliarden $\mathrm{CHF}^{26}$, was einer Kostensteigerung von $45 \%$ gegenüber der UKB entspricht (Abb. 22).

Als Überschreitungsursachen sind zu nennen (Abb. 22):

- Sicherheit und Stand der Technik

- Verbesserungen für Bevölkerung und Umwelt

- politisch und rechtlich begründete Verzögerungen

- Geologie

- Vergabe und Bauausführung

- Projekterweiterung

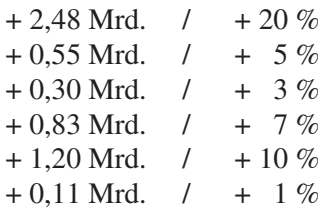

Daraus ist erkennbar, dass es sich bei den Mehrkosten mehrheitlich um Mehrinvestitionen und damit um Mehrbestellungen des Bundes handelt (26\% von total $45 \%$ ).

Der erreichte Wert von 17,65 Milliarden CHF (Preisbasis 1998) entspricht unter Berücksichtigung der unterschiedlichen Projektperimeter in den Jahren 1989 und 1998 ziemlich genau dem Wert der oberen Bandbreite aus Parlamentsbotschaft vom 23. Mai 1990 (10,1 Milliarden $+40 \%$, Preisbasis 1988). Die 17.695 Milliarden CHF entsprechen unter Anwendung der dem Projekt zu Grunde gelegten Teuerungsumrechnungen (NEAT Teuerungsindex von 105,9 für die Periode 1991 bis 1998 und Index des Zürcher Wohnbauindex von 120,427 für die Zeit 1988 bis 1991) einem Wert 13,88 Milliarden CHF (Abb. 23). Unter Berücksichtigung der Prognosebandbreite (die hinterlegten Risiken sind nachweisbar eingetreten) kann man von einer finanziellen Punktlandung am oberen Ende der Bandbreite sprechen, welche aber durch den Parlamentskredit aus dem Jahr 1990 abgedeckt war. Wie in diesem Beitrag gezeigt wurde, konnte mit dem gesprochenen Kredit zwar nicht das gesamte ursprünglich angedachte System gebaut werden. Der reduzierte Ausbau hatte aber

\footnotetext{
${ }^{26}$ Schweizerische Eidgenossenschaft, Oberaufsicht über den Bau der Neat in den Jahren 2018 und 2019, Bericht der Neat-Aufsichtsdelegation der eidgenössischen Räte, Bern, 4. November 2019.

${ }^{27}$ Mangels anderer Vergleichsgrössen damals beigezogene Teuerungsumrechnung.
} 
Änderungen + 5,506 Mrd. (+45\%)

- Leistungsbereinigte Kostenbezugsbasis 1998 (12,189 Mio. / 100\%)

asicherheit und Stand der Technik (+2479 Mio. $/+20 \%)$

$\square$ Verbesserungen fär Bevölkerung und Umwelt (+545 Mio. $/+4 \%$ )

$\square$ Politisch und rechtlich begründete Verzögerungen (+305 Mio. / +3\%)

$\square$ Geologie (+831 Mio. $/+7 \%)$

$\square$ Vergabe und Bauausführung (+1229 Mio. $/+10 \%)$

口Projekterweiterung (+106 Mio. / +1\%)

口Kostenpositionen BAV (+11 Mio. $/+0 \%$ )
Kostenprognose BAV 30.06.2019 $=17,695$ Mrd.

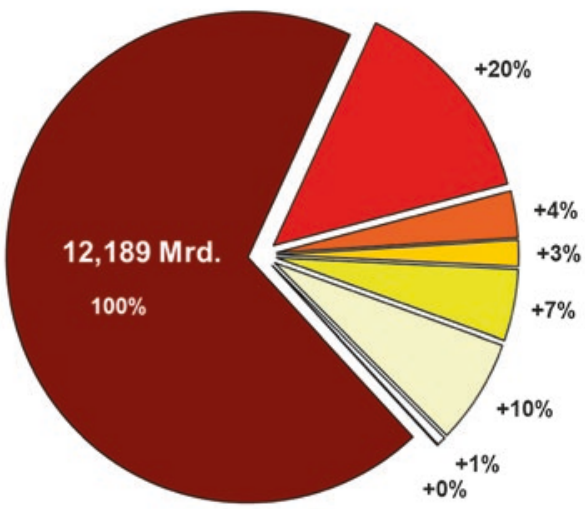

Abb. 22 Vergleich der Kostenbezugsbasis 1998 mit den prognostizierten Endkosten. (Quelle ${ }^{30}$ )

Eintretens-

wahrscheinlichkeit

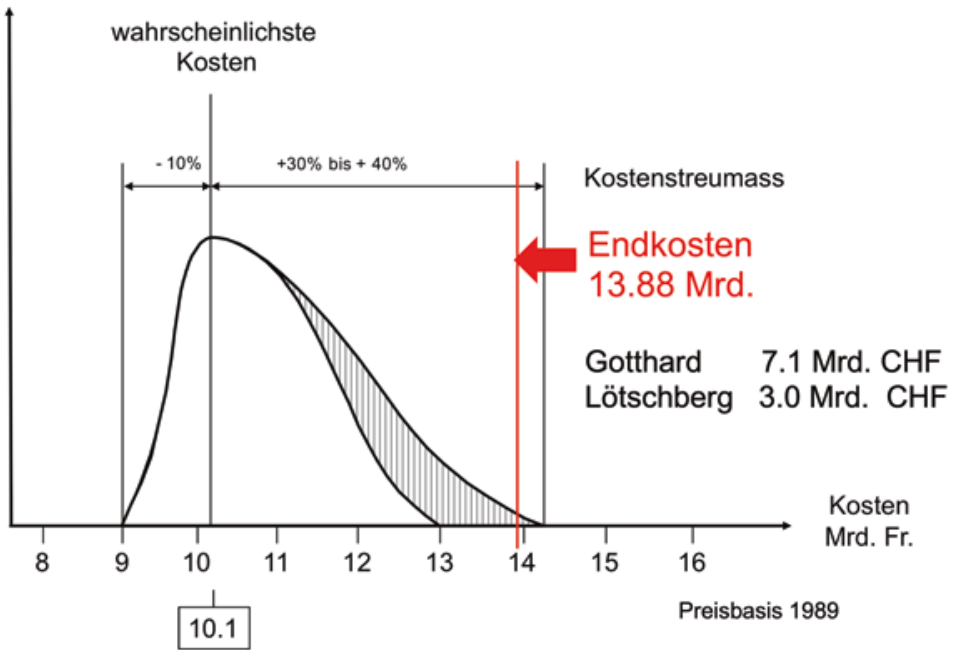

Abb. 231990 prognostizierte Endkosten der NEAT (Schweizerischer Bundesrat, Botschaft über den Bau der schweizerischen Eisenbahn-Alpentransversale (Alpentransit-Beschluss) vom 23. Mai 1990.) im Vergleich zu den effektiven Endkosten per Juni 2019 (auf die Preisbasis 1989 zurückgerechnet) 
bei den Kapazitäten nur geringe und bei den Fahrzeiten immer noch vertretbare Zielabweichungen zur Folge.

Diese These der stabilen Kosten bestätigt sich auch unter Berücksichtigung der mittlerweile sehr stabilen Endwertprognose, wonach sich die NEAT-Kosten zu aktuellen Preisen auf 22,75 Milliarden CHF belaufen, ein Wert, der geringfügig unter dem in der Parlamentsdebatte 1991 genannten Wert von 24 Milliarden (prognostizierte Baukosten, inkl. Risiken plus Teuerung und Zinskosten) liegt. ${ }^{28}$ Auch das ist ein Indikator für eine Punktlandung.

\section{Gesamtbeurteilung und Schlussfolgerungen}

Mit den drei Basistunneln am Lötschberg, Gotthard und Ceneri wurde innerhalb des vorgesehenen Zeit- und Kostenrahmens eine Eisenbahninfrastruktur geschaffen, welche es ab heute erlaubt, die versprochenen, wesentlich höheren Gütermengen auf der Schiene durch die Schweizer Alpen zu transportieren.

Dieser Erfolg kam nur dadurch zustande, weil Alle von Anfang mit viel Respekt an die Bewältigung der Aufgaben gingen, großmehrheitlich die richtigen Leute an den richtigen Stellen waren, welche wiederum Lösungen auf Basis der Kooperation und nicht der Konfrontation suchten. Zusätzlich ist zu erwähnen, dass das Projekt sehr sorgfältig von den besten und nicht den billigsten Auftragnehmern vorbereitet und ausgeführt wurde. ${ }^{29}$

Die mit diesen Leistungen geschaffenen Zusatzkapazitäten werden derzeit nur in geringem Ausmass genutzt, wobei es sich primär um Verkehr handelt, welcher sich von der Strasse auf die Schiene verlagert. Der angestrebte Trend zur Veränderung des Modal Splits zwischen Strasse und Schiene zu Gunsten der Schiene ist derzeit zu beobachten, auch wenn der Prozess eher langsam vonstattengeht.

Trotz erheblich erhöhter Schienentransportkapazitäten ergibt sich seit 2008 keine markante Steigerung des Gütertransportvolumens durch die Schweiz. Diese Entwicklung steht im Kontrast zur Verkehrsentwicklung an der Brennerachse wo die Transportmengen von 2008 mittlerweile markant überstiegen werden, primär aber mit einer Zunahme des Strassenverkehrs.

$\mathrm{Ob}$ sich der Verlagerungstrend in der Schweiz ab 2021 beschleunigen und die Transportmengen durch die Schweiz nach der Eröffnung des Ceneri-Basistunnels und des 4-Meter-Korridors per Ende 2020 zunehmen werde, wird der Verlagerungsbericht im Herbst 2023 erstmals zeigen.

Damit allfällige Mehrmengen mit Eisenbahnzügen gefahren werden können, gilt es aber in den Ursprungsländern und in den Zielländern sowie auf den Zulauf-

\footnotetext{
${ }^{28}$ Schweizerische Eidgenossenschaft, Parlamentsdienste, Verhandlungsheft 90.040, Neue Eisenbahn-Alpentransversale (NEAT), 1991.

${ }^{29}$ Ehrbar, Heinz; Lehren und Lernen aus dem schweizerischen AlpTransit Projekt am Beispiel des Gotthard-Basistunnels; Sonderdruck zum 11. Grazer Baubetriebs- \& Baurechtsseminar, ETH Zürich, D-BAUG, 2020.
} 
strecken die entsprechenden Netz- und Terminalkapazitäten zu schaffen. Damit ist sicherzustellen, dass die gewünschte Anzahl der Züge mit der erforderlichen Pünktlichkeit gefahren werden können. Unter Berücksichtigung der langen Vorbereitungs- und Realisierungszeiten sind die entsprechenden Massnahmen unverzüglich anzugehen und umzusetzen. Wird dies nicht getan, wird man in rund 10 Jahren ähnliche Feststellungen wie in der Schweiz auch an der Brennerachse tätigen müssen, dass die Infrastruktur für umweltfreundlichere, qualitativ hochstehende Transporte vorhanden ist, diese aber mangels rechtzeitig umgesetzter Begleitmassnahmen nicht umfassend genutzt werden kann.

Die Politik auf der europäischen und der nationalen Ebene der betroffenen Länder ist aufgefordert, die angedachten und teilweise schon vorhandenen Planungen, z. B. im Rahmen des Bundesverkehrswegeplans 2030 in Deutschland zügig und nachhaltig umzusetzen, damit die bereits in den Achtziger-Jahren angedachten strategischen Ziele zur Verlagerung des Güterverkehrs von der Strasse auf die Schiene auch erreicht werden können (Abb. 5).

Die derzeitige Verkehrsentwicklung, insbesondere in den Nachbarländern der Schweiz, ist derzeit weit von diesen Zielen entfernt (Abb. 24). Wie in diesem Beitrag gezeigt wurde, wäre die Schweiz mit einer Schienentransportkapazität von 67 Mio. Tonnen, von denen im Jahr 2018 deren 27,9 Mio. Tonnen genutzt wurden, aktuell in der Lage praktisch den gesamten Strassenverkehr am Brenner über die Schiene zu transportieren. Das wird nicht geschehen, weil sich die die Güterverkehrsströme nicht per Knopfdruck verlagern lassen. Dazu bräuchte es entsprechende Massnahmen bei den nördlichen Zulaufstrecken und bei den Terminalkapazitäten, um nur einen Teil des Verkehrs umzulenken. Ob sich dieser Effekt mit der Eröffnung des 4 m-Korridors durch die Schweiz per Ende 2020 einstellen wird, muss sich noch

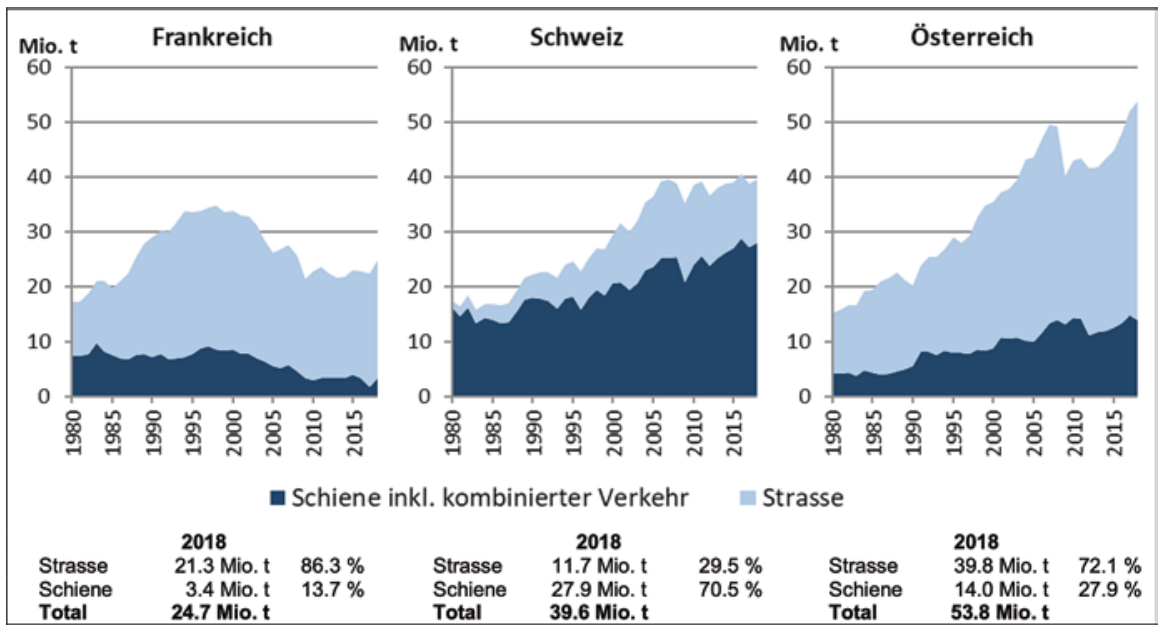

Abb. 24 Entwicklung des alpenquerenden Güterverkehrs seit 1980. Quelle (Schweizerische Eidgenossenschaft, Bericht des Bundesrats, Bericht über die Verkehrsverlagerung vom November 2019, Verlagerungsbericht Juli 2017-Juni 2019.) 


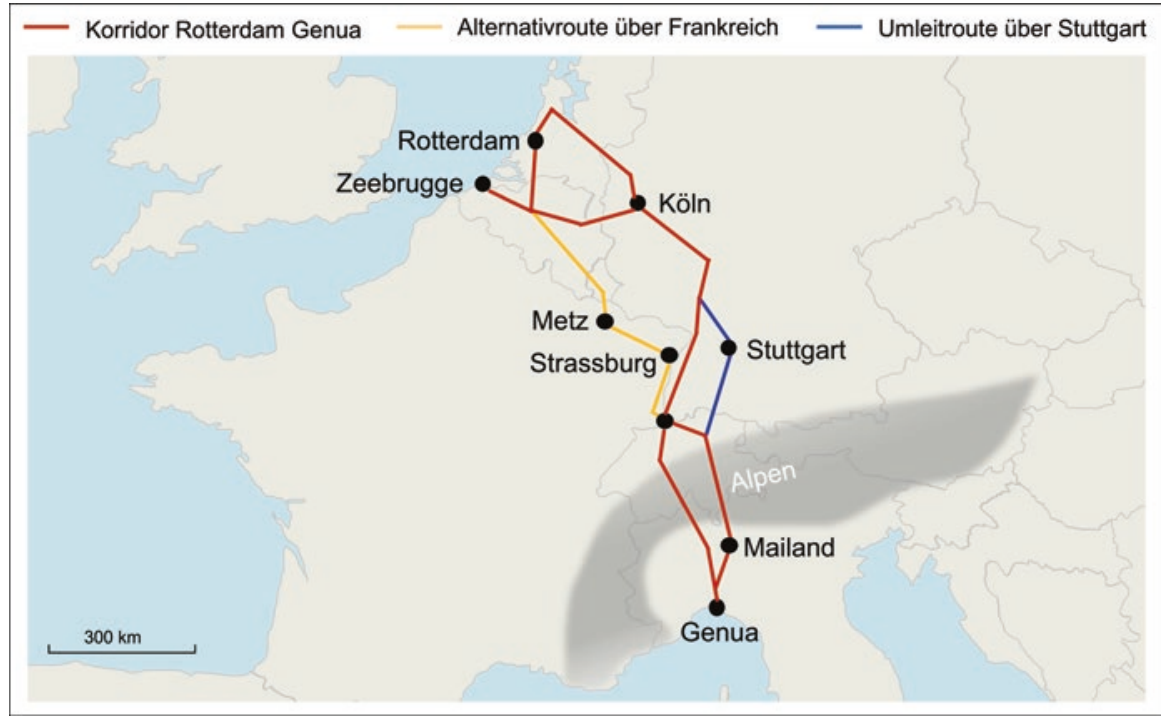

Abb. 25 Nördlicher NEAT-Zulauf: Vorgeschlagene Alternativrouten zur deutschen Rheintalstrecke (Bild basierend auf ${ }^{38}$ )

weisen. Eine gesunde Skepsis ist, wegen der fehlenden Kapazitäten im nördlichen Zulauf, auch ohne Effekte aus der Corona Krise, angezeigt. In Anbetracht der unsicheren Entwicklung in Deutschland, hat das schweizerische Parlament deshalb im Juni 2020 den Schweizerischen Bundesrat beauftragt, eine linksrheinische Alternativroute zu forcieren. Die Schweiz soll mit Frankreich und Belgien einen Staatsvertrag aushandeln, um auf der Route Basel-Strassburg-Metz an die Kanalküste einen Vier-Meter-Korridor zu realisieren ${ }^{30}$ (Abb. 25).

Das europäische verkehrspolitische Umfeld wäre gut beraten die entsprechenden Systemüberlegungen auf allen TEN-T Korridoren rasch voranzutreiben, damit mindestens bei der Eröffnung des Brenner-Basistunnels die Zweifel ausgeräumt sind, ob die Infrastruktur auch im erforderlichen Ausmass genutzt werden kann. Die derzeitigen Entwicklungen bei den Zulaufstrecken und beim politischen Willen zur Verlagerung des Güterverkehrs auf der Strasse auf die Schiene lassen vermuten, dass sich, wie im Fall der NEAT, ohne entsprechende Begleitmassnahmen auch am Brenner nicht von Anfang an der erwünschte Effekt in der Nutzung der Infrastruktur einstellen wird, zum Wohle der Umwelt und der betroffenen Regionen.

Die Geschichte scheint sich nach 25 Jahren zu wiederholen. 1995 schrieb Coopers \& Lybrand im Bericht ${ }^{31}$ zur NEAT-Finanzierung:

„Our analysis has shown that at the levels of prices recently charged for road and rail transport throughout Europe (assuming that Swiss rail prices can be restored to the levels

\footnotetext{
${ }^{30}$ Stalder, Helmut, Die Schweiz soll die Neat-Zufahrt in Frankreich mitfinanzieren, NZZ vom 20.08.2020.

${ }^{31}$ Coopers \& Lybrand, Financial review of the Neue Alpen-Transversale project, February 1995.
} 
assumed in the Message) the scheme is unlikely to be able to pay for itself within the time period of 70 years which has been set by the Swiss government. If the NEAT scheme is to meet the financial criteria it will therefore be necessary to adjust the prices charged for NEAT so that the revenue generated from users of the tunnels is sufficient to pay for the unusual levels of investment required to provide capacity for trans-alpine traffic.

This will not happen if the present policies towards the pricing of road and rail transport in Europe are maintained. ${ }^{32 ~ “ ~}$

Es bleibt also noch Vieles zu tun, nicht nur beim Tunnelbau, sondern auch beim Schaffen der verkehrspolitischen Randbedingungen und bei der noch fehlenden Infrastruktur im Gesamtsystem. Dazu braucht es den politischen Willen die entsprechenden Verladekapazitäten nördlich und südlich der Alpen zu schaffen, die Zulaufstrecken zeitgerecht entsprechend auszubauen und Anreize zu schaffen, dass die Verlagerung auch tatsächlich stattfindet, sei es durch eine hervorragende Transportqualität auf der Schiene oder aber allfälliger Steuerungsmassnahmen zur Schaffung einer Kostenwahrheit unter den Verkehrsträgern.

Sowohl für die AlpTransit-Strecken durch die Schweiz als auch an der BrennerAchse sind somit im internationalen Verbund noch längst nicht alle Hausaufgaben, gemacht, obwohl viel Zeit dazu vorhanden gewesen wäre.

Open Access Dieses Kapitel wird unter der Creative Commons Namensnennung 4.0 International Lizenz (http://creativecommons.org/licenses/by/4.0/deed.de) veröffentlicht, welche die Nutzung, Vervielfältigung, Bearbeitung, Verbreitung und Wiedergabe in jeglichem Medium und Format erlaubt, sofern Sie den/die ursprünglichen Autor(en) und die Quelle ordnungsgemäß nennen, einen Link zur Creative Commons Lizenz beifügen und angeben, ob Änderungen vorgenommen wurden.

Die in diesem Kapitel enthaltenen Bilder und sonstiges Drittmaterial unterliegen ebenfalls der genannten Creative Commons Lizenz, sofern sich aus der Abbildungslegende nichts anderes ergibt. Sofern das betreffende Material nicht unter der genannten Creative Commons Lizenz steht und die betreffende Handlung nicht nach gesetzlichen Vorschriften erlaubt ist, ist für die oben aufgeführten Weiterverwendungen des Materials die Einwilligung des jeweiligen Rechteinhabers einzuholen.

\footnotetext{
${ }^{32}$ Hervorhebung durch den Autor.
} 